\title{
Unidirectional recruitment relations between proteins can be determined by a CRISPR/dCas9 recruitment assay
}

6 Ido Lavi”, Supriya Bhattacharya ${ }^{\sharp}$, Ola Orgil, Nir Avital, Guy Journo, Vyacheslav Gurevich and Meir

7 Shamay*

8 Daniella Lee Casper Laboratory in Viral Oncology, Azrieli Faculty of Medicine, Bar-Ilan University, Safed, 9 Israel 1311502

10

$11{ }^{\#}$ These authors contributed equally to this work

$12 \quad{ }^{*}$ Corresponding author

13 Meir Shamay

14 Email: meir.shamay@biu.ac.il

15 Running Title: CRISPR/dCas9 recruitment assay

16 Author Contributions: Conceived and designed the experiments: I.L., S.B., M.S. Performed the 17 experiments: I.L., S.B., Analyzed the data: I.L., S.B., G.J. \& M.S. Generated tools \& reagents: O.O., 18 N.A., V.G., S.B., Wrote the paper: M.S.

19 Competing Interest Statement: The authors declare no competing financial interests.

20 Keywords: CRISPR/Cas9, dCas9, KSHV, HHV-8, LANA, MeCP2, Recruitment. 
Abstract

24 Directional recruitment of protein complexes is critical for proper function of many nuclear processes. Here we present CRISPR-PITA (Protein Interaction and Telomere Recruitment Assay), an assay that determines the ability of a given protein to recruit any other nuclear factor. The protein of interest is directed via CRISPR/dCas9, a dead Cas9 that does not cut DNA, to a repeat sequence, such as telomeres, to obtain dots that are easily detectable by microscopy. The recruitment of endogenous nuclear proteins to these dots can then be visualized using specific antibodies. We determined recruitment abilities in CRISPR-PITA to methyl-CpG binding protein MeCP2, histone deacetylase 1 (HDAC1), heterochromatin protein 1 (HP1 $\alpha$ ), and the latency-associated nuclear antigen (LANA) encoded by Kaposi's sarcoma associated herpesvirus (KSHV, HHV-8). LANA was able to recruit its known interactors ORC2 and SIN3A to LANA-telomere dots. In contrast, LANA was unable to recruit MeCP2 whereas MeCP2 was able to recruit LANA. Similarly, HDAC1 that interacts with MeCP2 through the transcriptional-repression domain (TRD) same as LANA, was unable to recruit MeCP2, but MeCP2 recruited HDAC1. One important function of LANA is to tether the viral episomal genomes to the cellular chromosomes during cell division. The unidirectional recruitment of LANA by $\mathrm{MeCP} 2$, makes $\mathrm{MeCP} 2$ a candidate anchor for $\mathrm{KSHV}$ genome tethering by LANA. We found that cells derived from Rett syndrome and express a mutant MeCP2 (T158M), impaired applicable protein recruitment assay based on CRISPR/dCas9.

\section{Significance Statement}

42 CRISPR/Cas9 is a revolutionary system that has profoundly impacted biology research. Here we present 43 another application for CRISPR/Cas9, in evaluating recruitment relations between nuclear proteins. A 44 protein of interest is directed to a repeat sequence via the catalytically inactive Cas9 (dCas9) to generate 45 easily detectable dots. Then, the recruitment of other nuclear proteins to these dots can be evaluated. Using 46 this assay, we show that some interacting proteins have a unidirectional recruitment property, where only 47 one of the proteins can recruit its partner. We propose that available interacting domains can force this 48 unidirectional recruitment. Using this recruitment assay, we found unidirectional recruitment of the KSHV encoded LANA and HDAC1 by MeCP2. Furthermore, this unidirectional recruitment is critical for viral latency, since LANA fails to maintain the viral genomes in MeCP2 mutant cells. 


\section{Main Text}

\section{Introduction}

For many nuclear processes directional recruitment of protein complexes is critical for proper function. Transcription factor that binds a specific DNA sequence recruits co-activators and general transcription factors that results in subsequent recruitment of RNA polymerase leading to gene expression. In this scenario, it is expected that a transcription factor of interest will recruit the co-activator. However, the coactivator that is recruited by other transcription factors as well, should not recruit the transcription factor of interest. One example of recruitment directionality is the estrogen receptor, where the combination of ligand binding, dimerization and sequence specific DNA binding are required to generate high affinity surface to recruit co-activator proteins (1). This conformational change upon DNA binding and dimerization will favor binding and recruitment of co-activator to enhancer/promoter bound receptor and minimize interaction with DNA-free receptor. Another example is the enhanceosome, where coordinated activation of three distinct transcription factors and the architectural protein HMG I(Y) bind cooperatively to interferon-beta enhancer (2). Together they form high affinity surface to efficiently recruit the co-activator CBP (3). Therefore, an assay that can determine recruitment relations between interacting proteins may provide deeper understanding of biological systems. Although Chromatin immunoprecipitation (ChIP) assays may assist in this regard, there is a lack of an easy recruitment assay.

$\mathrm{MeCP} 2$ binds methylated DNA and recruits co-repressor complexes to execute the readout of $\mathrm{CpG}$ methylation into transcription repression (4). Several studies detected interaction between MeCP2 and histone deacetylase 1 (HDAC1), and recruitment of histone deacetylase activity is important for transcription repression imposed by $\mathrm{MeCP} 2(5,6) . \mathrm{MeCP} 2$ and $\mathrm{HDAC} 1$ represent an example of two proteins that interact in the nucleus. While $\mathrm{MeCP} 2$ recruits $\mathrm{HDAC} 1$ to methylated regions in order to repress transcription, we expect that HDAC1 should not recruit MeCP2 to promoters in those cases where HDAC1 is recruited by other transcription factors. Another protein that plays important role in transcription repression is heterochromatin protein 1 (HP1). HP1 interacts with the repressive mark histone 3 lysine 9 tri-methylation (H3K9Me3) and via recruitment of SUV39H1, the histone H3K9Me3 methyltransferase, leads to spreading of this heterochromatin repressive histone mark $(7,8)$. HP1 has been shown to interact with $\mathrm{MeCP} 2(9,10)$, but whether HP1 can recruit MeCP2 is an open question.

Kaposi's sarcoma associated herpesvirus (KSHV, HHV-8) is associated with several human malignancies $(11,12)$. Like all herpes viruses KSHV has two phases: latent (dormant) and lytic (productive) cycle. During latency the viral genomes reside in the nucleus of infected cells as large non-integrated plasmids, known as 
viral episomes. All KSHV infected cells express LANA, and LANA is essential for viral latency (13-15). LANA binding to the viral episomes is critical both for replication by recruitment of the cellular replication machinery to the viral genomes $(16,17)$, and for maintenance by tethering the viral episomes to the cell chromosomes during cell division $(18,19)$. Multiple LANA binding sites (LBS1 and LBS2) $(20,21)$ within the KSHV terminal repeats, and its oligomerization ability $(22,23)$ create clearly visible LANA dots in KSHV-infected cells. In contrast, LANA is equally distributed in the nucleus when it is expressed in uninfected cells. So, it is challenging to determine whether LANA or the viral genomes are the recruiters of cellular factors.

Cas9 is an endonuclease that can be directed to different DNA targets that are complimentary to a guide RNA and contain a PAM sequence (24). Soon after the discovery of this property, many applications of this sequence specific targeting of Cas9 emerged. For targeting purposes, the endonuclease is no longer needed, and a catalytically dead mutant Cas9 (dCas9) that still retains DNA binding capability was created $(24,25)$. Targeting this dCas9 to promoter sequences results in transcription activation or repression depending on the fused domains and the targeting location relative to the transcription start site $(25,26)$. Shortly after the creation of the dCas9 it was directed to repeat elements for the visualization of these elements in fixed (27) and live cells $(28,29)$. Targeting dCas9 to telomeres was also applied in an in-vivo mouse model (30).

101

Here we present a simple recruitment assay based on CRISPR/Cas9. We combined the ability of the SunTag system (31) to gather up to ten molecules of a protein of interest with CRISPR/dCas9 targeted to a repeat sequence such as telomeres. The ability of this protein to recruit endogenous or fluorescently tagged proteins to these telomere dots is determined. Interestingly, we find that the recruitment does not always work bi-directionally. LANA fails to recruit $\mathrm{MeCP} 2$, but $\mathrm{MeCP} 2$ efficiently recruits LANA. The unidirectional recruitment of LANA by $\mathrm{MeCP} 2$ raised the possibility that $\mathrm{MeCP} 2$ may serve as a protein anchor for LANA tethering. Indeed, we found that the virus fails to maintain its episomes in cells expressing a mutant MeCP2. We also investigated the recruitment relations between MeCP2 and HDAC1, and found that $\mathrm{MeCP} 2$ can recruit HDAC1, but HDAC1 is unable to recruit MeCP2. While both LANA and HDAC1 interact with the transcriptional-repression domain (TRD) of MeCP2, heterochromatin protein $1(\mathrm{HP} 1 \alpha)$ interacts the N-terminal domain (amino acids 1-55) of MeCP2 (9). Interestingly, we found that $\mathrm{HP} 1 \alpha$ is

112 able to recruit MeCP2. In summary, CRISPR-PITA can determine recruitment relations between proteins 113 and highlight the critical role of available interaction domains to determine recruitment directionality. 


\section{Results}

\section{Creation of visible dots at the telomeres with dCas9-SunTag and Fc-LANA}

The ability of dCas9 to direct proteins of interest to any genomic locus makes it an ideal system for a recruitment assay. Previous studies used the dCas9 system for labeling locus specific regions and targeting dCas9 to repeat genomic regions generated visible dots in fixed and live cells (27-29). In order to enhance dot formation, we utilized the SunTag system where tandem epitopes can concentrate multiple molecules of an antibody-fused protein that recognizes this epitope (up-to 10 with the tag used in this study) (31). In our case the tandem epitopes are fused to dCas9 and the antibody Fc is fused to our protein of interest (Fig. 1A).

For a proof of concept, we used KSHV encoded LANA that localizes in large dots together with the viral episomes in KSHV infected cells but is equally distributed in the nucleus when expressed in un-infected cells (32). Transfection of LANA fused with the antibody Fc (Fc-LANA) together with dCas9 and sgRNA for telomere repeat sequence, resulted in LANA-telomere-dots that were visible by immuno-fluorescent assay (IFA) for LANA (Fig. 1B). When the dCas9 was omitted from the mix LANA could not generate nuclear dots, suggesting that LANA-telomere-dots are dependent on the targeting of LANA to telomeres via dCas9. To validate that LANA was targeted to the telomeres we used an antibody against the telomeric repeat factor 2 (TRF2), and indeed Fc-LANA co-localized with TRF2 when co-transfected with dCas9 and sgTelomere (Fig. S1A). The larger dots of TRF2 observed in cells where LANA is targeted to telomeres via dCas9-SunTag, can be explained by LANA's ability to oligomerize and cluster several viral genomes $(20,22,23,33-35)$. Studies of the LANA oligomerization property identified a LANA oligomerization mutant $(22,23)$. Therefore, we created this LANA oligomerization mutant (F1037A/F1041A) in the context of Fc-LANA (Fc-LANA-mutant) and compared the dot size to Fc-LANA. As expected, the LANA oligomerization mutant created smaller telomere dots compared to wt LANA (Fig. S1B), indicating that targeting to telomeres can also determine oligomerization ability and identify oligomerization mutants. Altogether, these experiments indicate that we can generate LANA dots in un-infected cells using the dCas9 SunTag system.

\section{LANA recruits ORC2 and SIN3A but not MeCP2}

In this assay a protein of interest is targeted to telomeres via dCas9, and the recruitment of endogenous proteins to these dots is detected by immunofluorescence assay (Fig. 1C). We termed this method CRISPRPITA for Protein Interaction and Telomere recruitment Assay. We performed the CRISPR-PITA assay for ORC2, a subunit of the Origin Replication Complex (ORC), previously reported to interact with LANA 
and localize with LANA-episome-dots $(23,36-38)$. ORC2 was recruited by LANA, as was determined by the easily detected dots that co-localized with LANA-telomere-dots (Fig. 2A). It is important to note, that while the dCas9 and Fc-LANA were ectopically expressed, the recruited proteins are endogenous proteins, indicating the ability of LANA to recruit the endogenous ORC2. To quantify recruitment in unbiased manner, we created a measurement tool for dot formation. Using ImageJ the number of large dots and small dots of the analyzed protein in each cell are counted and then the ratio between large and small dots for each cell is calculated. The average of the ratios measured in several individual cells $(n=15)$ is presented. This measurement (average [large/small dots]) clearly shows that ORC2 was recruited to LANA-telomeredots.

Several studies reported on interaction between LANA and the corepressor protein SIN3A (mSin3a) (38, 39), and the methyl-CpG binding protein MeCP2 (40-42), but it was reported that MeCP2 is not associated with LANA dots in KSHV infected cells (43). We found that Fc-LANA recruited SIN3A to LANAtelomere-dots, both by visual observation and by our quantification (average [large/small dots]) (Fig. 2B). Analysis of MeCP2 recruitment via the CRISPR-PITA revealed no recruitment of MeCP2 to LANAtelomere-dots (Fig. 2C). Co-immunoprecipitation assay indicated that Fc-LANA was able to immunoprecipitate MeCP2 (Fig. S2), excluding the possibility that the lack of recruitment is due to the fusion between $\mathrm{Fc}$ and LANA that disrupted the interaction between LANA and MeCP2. Another possibility to explain the lack of $\mathrm{MeCP} 2$ recruitment by LANA, is that as a methylated DNA binding protein, it is engaged in binding methylated DNA and therefore not available to be recruited to LANAtelomere-dots. To test this, we performed the CRISPR-PITA in cells with knock-out (KO) of DNMT1 and DNMT3b (HCT DKO) that were reported to lose over 95\% of their CpG methylation (44). Even in HCT DKO, Fc-LANA could not recruit MeCP2 to LANA-telomere-dots (Fig. S3), suggesting that protein availability could not explain the lack of recruitment in this case. Immunofluorescence assays for ORC2, SIN3A and MeCP2 in KSHV-infected PEL (BCBL1) cells, revealed that while ORC2 and SIN3A were found associated with LANA-episome-dots, MeCP2 was not associated with the LANA-episome-dots (Fig. S4), supporting the ability of the CRISPR-PITA to predict recruitment ability between proteins.

\section{MeCP2 recruits LANA}

The observation that Fc-LANA could not recruit MeCP2, despite their documented interaction (40-42) and co-immunoprecipitation performed in this study (Fig. S2), highlights the importance to determine recruitment relations between proteins. Krithivas et al. have shown that expression of human MeCP2 is able to recruit LANA to heterochromatin in NIH 3 T3 mouse cells (40). Therefore, we tested the ability of 
Fc-MeCP2 to recruit LANA to MeCP2-telomere-dots. Cells were co-transfected with dCas9, sgTelomere, $\mathrm{Fc}-\mathrm{MeCP} 2$ and expression vectors for GFP-LANA or GFP alone as control. Fc-MeCP2 was able to recruit GFP-LANA to MeCP2-telomere-dots, but not GFP alone, supporting the ability of MeCP2 to recruit LANA (Fig. 3A). LANA contains three distinct domains, the N-terminal region (AA 1-329), the C-terminal (AA 936-1162), and a middle repeat region. Both the $\mathrm{N}$ and C-terminal regions have been shown to mediate chromosome association; the $\mathrm{N}$ via binding to core histones $\mathrm{H} 2 \mathrm{~A}$ and $\mathrm{H} 2 \mathrm{~B}$ (45), while for the C-terminal several candidates were identified including MeCP2, DEK and RING3 $(40,41,46)$. To determine the region of LANA sufficient for recruitment by MeCP2, we performed CRISPR-PITA by Fc-MeCP2 for LANA $\mathrm{N}+\mathrm{C}$, LANA $\mathrm{N}$ and LANA C. While both LANA N+C and LANA C were efficiently recruited by MeCP2, no recruitment was observed with the N-terminal (Fig. 3B). This result is in agreement with a previous study that located the interaction between LANA and MeCP2 to the C-terminal region (41). This result indicates that despite the high concentration of the recruiter protein at telomeres, still CRISPR-PITA requires specific interaction, as both the N-terminal of LANA and GFP-alone were not recruited by MeCP2 in our assay. This set of experiments highlights the distinction between interaction and recruitment, while LANA and MeCP2 were shown to interact with each other, still MeCP2 can recruit LANA, but LANA cannot recruit $\mathrm{MeCP} 2$.

\section{MeCP2 is able to recruit HDAC1, but HDAC1 is unable to recruit MeCP2}

Several studies detected interaction between MeCP2 and histone deacetylase 1 (HDAC1), and this recruitment of histone deacetylase activity is important for transcription repression imposed by MeCP2 (5, 6). MeCP2 and HDAC1 represent an example where two proteins can interact to form protein complexes in the nucleus. We expect that MeCP2 should recruit HDAC1 to methylated regions in order to repress transcription, but HDAC1 should not recruit MeCP2 to the promoters where it is recruited by other transcription factors. Therefore, we applied the CRISPR-PITA to determine recruitment relations between these two cellular proteins. CRISPR-PITA revealed that Fc-MeCP2 efficiently recruits HDAC1 to MeCP2telomere-dots (Fig. 4A). In contrast, Fc-HDAC1 failed to recruit MeCP2 to HDAC1-telomere-dots both in HCT (Fig. 4B) and HCT DKO cells (Fig. 4C). Thus, in the context of MeCP2 and HDAC1, recruitment is also unidirectional, enabling $\mathrm{MeCP} 2$ to recruit $\mathrm{HDAC} 1$ to repress transcription, but preventing the mislocalization of MeCP2 by HDAC1.

\section{Heterochromatin protein 1 is able to recruit $\mathrm{MeCP} 2$}

Both LANA and HDAC1 interact with the same region of $\mathrm{MeCP} 2$, the transcriptional-repression domain (TRD), that is located next to the methyl-CpG-binding domain (MBD) (35, 40). In contrast, 
Heterochromatin protein 1 (HP1 $\alpha$ ) interacts with the N-terminal domain (amino acids 1-55) of MeCP2 (9). We hypothesized that since HP1 $\alpha$ interacts with a different domain that might be available for interaction also in its DNA-free state, HP1 $\alpha$ might be able to recruit MeCP2. Interestingly, in CRISPR-PITA we found that Fc-HP1 $\alpha$ was able to recruit MeCP2 (Fig. 5). This result supports the notion that available interacting domains determine recruitment directionality.

\section{Rett syndrome mutant MecP2 cannot support KSHV maintenance during latency}

One important function of LANA is to maintain the viral episomal genomes, by tethering the viral genomes to cellular chromosomes during cell division, ensuring they remain in the nucleus once nuclear envelope is reformed. To do so, LANA directly binds the LANA binding sites (LBS1 and LBS2) $(20,21)$ on the viral genomes, and associates with cellular chromosomes. Our finding that MeCP2 recruits LANA, but LANA cannot recruit $\mathrm{MeCP} 2$, suggests that the interaction with $\mathrm{MeCP} 2$ is permitted only once it is bound to methylated DNA, and therefore may be a potential candidate for LANA tethering. Sporadic mutations in MeCP2 are associated with most cases of Rett syndrome (RTT), a severe X-linked neurodevelopmental disorder in humans. (47). Lymphoblastoid Cell Lines (LCLs) derived from RTT patients expressing wildtype (wt) MeCP2 allele, mutant T158M (that cannot bind methylated DNA), and 803delG (deletion of the C' terminus) of MeCP2 (48) were tested for their ability to support KSHV episome maintenance. Cells were infected with rKSHV (rKSHV.219) expressing GFP and maintenance of the viral episomal DNA and the percentages of GFP positive cells were analyzed (Fig. 6 A \& B). While both wt and 803delG efficiently supported viral latency, the MeCP2 mutant in the MBD that cannot bind DNA (T158M) could not. This result indicates that a functional $\mathrm{MeCP} 2$ that is able to bind methylated DNA is essential for KSHV genome maintenance. LANA has been shown to bind both the MBD and TRD of MeCP2 (41). To determine if LANA can be recruited by the T158M mutant, we cloned it into Fc-MeCP2 and performed CRISPR-PITA assay. We found that MeCP2 T158M mutant can recruit LANA (although to a lesser extent), when it is artificially targeted to chromatin by dCas9 (Fig. 6C). MBD deletion mutant, partially lost the ability to recruit LANA when artificially targeted to chromatin. To assay for both abilities to bind LANA and to bind methylated DNA, we harnessed a specific feature of $\mathrm{MeCP} 2$ to localize to heterochromatin loci in mouse cells. This assay is typically performed in NIH $3 \mathrm{~T} 3$ cells, since they express very low levels of endogenous MeCP2 $(40,49)$. Similar to previous studies $(40,41)$, we found that LANA can be efficiently recruited to heterochromatin when co-transfected with MeCP2 (Fig. 6D). In contrast, both T158M and MBD-del mutants were not associated with heterochromatin, and therefore could not recruit LANA to heterochromatin loci. Altogether, we found that Rett syndrome mutant MeCP2 T158M, that cannot recruit 
LANA to methylated DNA, also cannot support KSHV episome maintenance. We revealed a critical role for MeCP2 in KSHV episome maintenance by LANA.

\section{Discussion}

A powerful method to study recruitment is the Chromatin immunoprecipitation (ChIP) assay, that can detect association of proteins with specific DNA sequences $(50,51)$. ChIP assays require fixation, proper shearing of the chromatin, and special antibodies that can perform the immunoprecipitation of the cross-linked chromatin. In many cases even when two or more proteins are associated with the same genomic region, it is hard to evaluate the relationship between the proteins and determine essential factors for the recruitment. Although this limitation can be overcome by using Knockout $(\mathrm{KO})$ or Knockdown (KD) of one protein and testing the ability of other proteins to be recruited to DNA/chromatin in the absence of that protein, this raises additional challenges in creating $\mathrm{KO}$ or $\mathrm{KD}$ of the target gene. The SOS-recruitment assay (SRA) can identify protein-protein interactions via recruitment of a "prey" protein by a membrane associated "bait" (52). This is a powerful method to identify protein-protein interactions, but the limitation of this method is that both the "prey" and the "bait" are ectopically expressed, and interaction takes place in the cytoplasm of yeast cells, which is less relevant for protein complexes in the nucleus. These assays most often express a random library with partial protein sequences, and therefore cannot predict the ability to recruit the full-length protein with its multiple protein-protein, protein-RNA or protein-DNA interactions that might alter its ability to be recruited. Another recruitment assay is based on integration of the lac $\mathrm{O}$ array into the genome and directing the protein of interest to this array via fusion to the Lac-repressor (53, 54). One limitation of this assay is the limited number of cell lines that were generated to contain integration of this $l a c \mathrm{O}$ array.

Here we describe a simple method to test for recruitment relations between proteins. This method does not require chromatin shearing and can be performed with various antibodies that can recognize the native form of proteins, and in the absence of such an antibody a recruitment of fluorescently labeled proteins can be observed. To obtain visible dots we combined the ability of the repeated epitope SunTag with dCas9 targeted to a repetitive genomic sequence such as the telomeres. Although targeting dCas 9 to telomeres has been performed previously to follow nuclear events such as, liquid-liquid phase separation (LLPS), or the generation of heterochromatin $(55,56)$, but has not been applied to test recruitment directionality. Using this method we found unidirectional recruitment relations between proteins. Our results with CRISPRPITA, indicated that LANA was able to recruit ORC2 and SIN3A, but was unable to recruit MeCP2 to LANA-telomere-dots. While it has been shown that ORC2 localizes with LANA dots $(23,36-38)$, and MeCP2 does not (43), there was no published data on SIN3A localization in KSHV infected cells. So, we 
performed immunofluorescence assay in KSHV-infected BCBL1 cells and found that both ORC2 and SIN3A were associated with LANA dots, but MeCP2 was not. These results are in agreement with CRISPRPITA, supporting the ability of CRISPR-PITA to predict recruitment ability of proteins. The recruitment of GFP-LANA by Fc-MeCP2 also suggests that CRISPR-PITA can be done without cell fixation or immunofluorescence, but directly via detection of fluorescently-labeled proteins. This property might be more relevant in cases where CRISPR-PITA is applied for screening purposes.

The combination of both the SunTag and a repeat element in targeting the protein of interest, results in high protein concentration at specific nuclear locations. One concern that might be raised is whether the recruitment we observed with CRISPR-PITA is specific or an artifact of the high protein concentration. To test this possibility, we performed the CRISPR-PITA with Fc-MeCP2 and LANA deletion mutants. We found that $\mathrm{Fc}-\mathrm{MeCP} 2$ recruited the $\mathrm{C}$-terminal of LANA, but not its $\mathrm{N}$-terminal. Interaction of LANA Cterminal domain with MeCP2 is in agreement with a previous study for LANA (41). The related gamma2 herpesvirus, herpesvirus saimiri (HVS) encodes for ORF73, a functional homolog of KSHV encoded LANA (also known as ORF73). Also HVS encoded ORF73 interacts with MeCP2 via its C-terminal domain (49). The lack of recruitment for the N-terminal domain of LANA, indicated that recruitment is still specific and requires the interaction domain. Another support for the specific recruitment by CRISPR-PITA is the lack of recruitment observed for GFP alone. A growing list of nuclear proteins with intrinsically disordered regions and RNA/DNA binding properties can promote liquid-liquid phase separation (LLPS); both LANA and $\mathrm{MeCP} 2$ possess this property $(57,58)$. Rett syndrome MeCP2 mutants, including T158M, are impaired in LLPS formation. Since T158M mutant still can recruit LANA in CRISPR-PITA, indicates that the recruitment we observed is not dependent on LLPS formation.

The inability of LANA to recruit MeCP2, despite their documented interaction prompted us to test whether MeCP2 can recruit LANA. Indeed, in CRISPR-PITA Fc-MeCP2 was able to recruit LANA. This result comes in agreement with a previous study where $\mathrm{MeCP} 2$ recruited LANA to mouse heterochromatin structures (40). This result also highlights the importance of a recruitment assay as recruitment is not always bi-directional, and in many cases should be functionally unidirectional. One of LANA functions where unidirectional recruitment seems essential, is LANA tethering of the viral episomal genome to the cell chromosomes during cell division. Therefore, we tested the possible role of MeCP2 in KSHV episome maintenance. We found that Rett syndrome mutant MeCP2, that cannot bind methylated DNA (T158M), could not support KSHV latency. Our result suggests that in addition to LANA interaction with histones (45), MeCP2 adds another layer to secure chromosome tethering (suggested model in Fig. 7). Similar to KSHV encoded LANA, HVS encoded ORF73 has a critical role in viral episomal genome maintenance 
(59). MeCP2 has been shown to be essential for tethering of the viral genomes by ORF73 to maintain HVS latency (49). Therefore, our observation that MeCP2 is essential for episome maintenance, seems to be conserved between KSHV and HVS. In this scenario LANA/ORF73 binds MeCP2 to anchor the viral episomes to cellular chromosomes. If the anchor (MeCP2) is free (not bound to DNA/chromosome), this may lead to viral loss. Therefore, in this case unidirectional recruitment is a matter of survival or extinction.

A nice example of directional recruitment is the case of MeCP2 that binds methylated DNA and recruits co-repressor complexes including $\mathrm{HDAC} 1(5,6)$. In this case we expect that MeCP2 should recruit HDAC1, leading to transcription repression. On the other hand, HDAC1 is recruited by many transcription factors to generate repressive chromatin. Therefore, we expect that it should not recruit MeCP2 to all the chromosome locations it is recruited by other transcription repressors. Indeed, we found that Fc-MeCP2 was able to recruit HDAC1 to MeCP2-telomere-dots, but Fc-HDAC1 was unable to recruit MeCP2. How come two proteins that interact with each other can generate unidirectional recruitment? $\mathrm{MeCP} 2$ is an intrinsically disordered protein that is monomeric in solution even at high concentrations but dimerizes upon DNA binding $(60,61)$. Both LANA and HDAC1 interact with the transcriptional-repression domain (TRD) of MeCP2 $(6,41)$ that is located next to the methyl-CpG-binding domain (MBD). In contrast, HP1 $\alpha$ interacts with the N-terminal domain (amino acids 1-55) of MeCP2 (9). Therefore, while the TRD domain should be available for interaction only upon DNA binding and $\mathrm{MeCP} 2$ dimerization, we predicted that the $\mathrm{N}$-terminal domain might be available for interaction also in the form of DNA-free MeCP2. Indeed, we found that $\mathrm{HP} 1 \alpha$ is able to recruit MeCP2 to HP1 $\alpha$-telomere-dots. Based on these characteristics and our CRISPR-PITA results we came to the following model; when MeCP2 is not associated with DNA it is a monomer and therefore the TRD is not available to interact and therefore cannot be recruited by HDAC1 and LANA. When MeCP2 binds methylated DNA its conformation changes so the TRD can bind and recruit HDAC1 and LANA. In this way, unidirectional recruitment of HDAC1 and LANA only to DNA bound MeCP2 is executed, while interaction with free MeCP2 is prevented (Fig. 7). On the contrary, $\mathrm{HP} 1 \alpha$ interacts with a different region, the $\mathrm{N}$-terminus of $\mathrm{MeCP} 2$ that is available for binding also in its DNAfree state, and therefore $\mathrm{HP} 1 \alpha$ can recruit MeCP2. It has been shown that phosphorylation at serine 229 (pS229) of MeCP2 strongly enhances the interaction between MeCP2 and HP1 $\alpha$ (10). Our observation that $\mathrm{HP} 1 \alpha$ can recruit MeCP2, may explain their observation that MeCP2 pS229 was enriched at the receptor tyrosine kinase gene RET promoter.

Here we present only few examples of unidirectional recruitment. There are many cellular functions that should be executed in a directional recruitment manner. The CRISPR-PITA we present in this study is a 
convenient tool to ask these questions and has the potential to push forward our understanding of many cellular functions.

\section{Materials and Methods}

Cell culture. SLK cells (kindly provided by Don Ganem \& Rolf Renne) and NIH 3T3 cells (kindly provided by Oren Kobiler) were cultured in Dulbecco's modified Eagle's medium (DMEM) supplemented with 10\% fetal bovine serum (heat inactivated) and $100 \mathrm{U} / \mathrm{ml}$ penicillin, $100 \mu \mathrm{g} / \mathrm{ml}$ streptomycin, $2 \mathrm{mM}$ L-glutamine, and $1 \mathrm{mM}$ Sodium-Pyruvate in $5 \% \mathrm{CO}_{2}$ at $37^{\circ} \mathrm{C}$. HCT and HCT Dnmt1-/- Dnmt3b -/- (DKO) cells (kindly provided by Bert Vogelstein) were grown in McCoy's 5A medium supplemented with the above-mentioned supplements. BJAB and BCBL1 cells (kindly provided by Richard F. Ambinder), and LCLs (kindly provided by Uta Francke), were cultured in RPMI-1640 medium supplemented with 20\% FBS and the above-mentioned supplements. Single-cell derived clonal LCLs expressing the wt or mutant Rett syndrome MeCP2 allele were established in the lab of Uta Francke (48).

Plasmids. pHRdSV40-dCas9-10xGCN4_v4-P2A-BFP (Addgene plasmid \# 60903; http://n2t.net/addgene:60903; RRID:Addgene_60903) and pHR-scFv-GCN4-sfGFP-GB1-dWPRE (Addgene plasmid \# 60907; http://n2t.net/addgene:60907; RRID:Addgene_60907) were a gift from Ron Vale (31). pgRNA-humanized was a gift from Stanley Qi (Addgene plasmid \# 44248; http://n2t.net/addgene:44248; RRID:Addgene_44248) (25). The sgRNA targeting telomeres (TTGGGTTAGGGTTAGGGTTAGGGTTAGTTTTAGAGCTAGAAATAGCAAGTTAAAATAAGGC TAGTCCGTTATCAACTTGAAAAAGTGGCACCGAGTCGGTGCTTTTTTC) was cloned into pgRNA-humanized. LANA, HDAC1, HP1 $\alpha$ and MeCP2 were PCR amplified and cloned into pHR-scFvGCN4-sfGFP-GB1-dWPRE. Fc-LANA was further subcloned into pLIX_403, a gift from David Root (Addgene plasmid \# 41395; http://n2t.net/addgene:41395; RRID:Addgene_41395). pEGFP-c2 from Clontech. GFP-LANA and LANA deletion plasmids were described previously $(38,62)$. For generation of MeCP2 (T158M) mutation RNA was isolated from LCL 487 (T158M) cell line by Qiagen RNeasy mini kit (cat no \# 79656) and cDNA was prepared by maxima H minus first strand cDNA synthesis kit (cat no \# K1682). A fragment of MeCP2 containing the T158M mutation was amplified by PCR and cloned in MeCP2-GFP plasmid (Addgene \# 48078) and subsequently cloned into pHR-scFv-GCN4-sfGFP-GB1dWPRE plasmid. MBD domain deleted MeCP2 was created by overlap extension PCR. Oligomerization mutant LANA was created by Q5 site directed mutagenesis kit (cat no\# NEB E0554S). All primers can be found in supplementary primer list (Table S1). 
Immunofluorescence assay. $1 \times 10^{5}$ cells were plated in 12 wells plate. Next day the cells were washed once with PBS and were transfected using PolyJet In Vitro DNA Transfection Reagent (SignaGene Laboratories Cat \# SL100688) with the appropriate plasmids. In the day after $4 \times 10^{4}$ cells were transferred into chamber slide cell culture glass 8 wells (SPL life Science LTD). Next day, the cells were washed once with PBS followed by fixation in 4\% paraformaldehyde in PBS pH 7.4 for 10 minutes. After 3 washes with PBS, cells were permeabilized for 10 min with PBS containing $0.25 \%$ Triton X-100. Cells were washed 3 times with PBS and blocked with 1\% BSA, $22.52 \mathrm{mg} / \mathrm{mL}$ glycine in PBST (PBS $+0.1 \%$ Tween 20) for 45 $\min$ in $37^{\circ} \mathrm{C}$. The slides were incubated for 1 hour with primary antibodies, followed by 3 washes with PBS. Then, the slides were incubated with secondary antibodies for 1 hour and washed again 3 times with PBS and mounted with Vectaschield containing DAPI (Vector Laboratories, H-1500).

Antibodies. The following antibodies were used for immunofluorescence studies: Rat anti LANA (Advanced Biotechnologies, cat\#13-210-100), Mouse anti LANA (Leica, NCL-HHV8-LNA), Mouse anti TRF2 (Merck, 05-521), Rabbit anti MeCP2 (Abcam, ab2828), Rabbit anti mSin3a/SIN3A (Abcam, ab3479), Mouse anti HA (Sigma H9658), Mouse anti ORC2 (MBL Life Science, M055-3), and Rabbit anti HDAC1 (Abcam, ab53091). Goat anti rabbit Alexa 488 (Abcam, ab150077), Goat anti Rat Alexa 488 (Abcam, ab150157), and Goat anti Mouse Alexa 594 (Abcam, ab150116). Fc-MeCP2, Fc-HP1 $\alpha$, and FcHDAC1 were detected with Mouse anti HA. Fc-LANA was detected by Mouse anti LANA, except in combination with TRF2 where Rat anti LANA was used. FLAG-LANA mutants $(\mathrm{N}+\mathrm{C}, \mathrm{N}, \mathrm{C})$ were detected with Rabbit anti FLAG (Sigma 7425).

Confocal imaging. Images were captured with Zeiss LSM780 inverted confocal microscope through a 63X objective with $\mathrm{Z}$ stack mode. Then, the middle stacks were selected and merged using Subset mode and Maximum Intensity Projection mode, respectively with Zenn software black edition. Processing and exporting of the resulted images was done with Zenn software blue edition.

Dot Quantification. Merged stacks images were analyzed with FIJI software as follow: Signal exposure of loaded images was set using the Threshold mode to the point that clear separated dots were seen, followed by Watershed mode, in order to fine tune the separation of the dots. Then, Region of Interest was selected, and measurements were directed to the appropriate channel. Area size in micron ${ }^{2}$ of the dots was collected using Analyze Particles mode for each and every selected cell.

LCL infection and maintenance assay. LCLs were infected with KSHV by co-culture (63). Briefly, VerorKSHV.219 cells (45) were induced with $1.25 \mathrm{mM}$ sodium butyrate and $20 \mathrm{ng} / \mathrm{ml}$ 12-Otetradecanoylphorbol-13-acetate (TPA) and after $48 \mathrm{~h}$ were cocultured with LCL cells (1:1 cell ratio) in 
RPMI 1640 medium for an additional $48 \mathrm{~h}$. Medium containing the LCLs infected cells was collected and transferred to a new flask for an additional $48 \mathrm{~h}$. Transferred again to a new flask, and at days $2,7,14,24$, cells were taken for FACS analysis to detect the number of GFP positive cells. At the same time points, genomic DNA was isolated from with DNeasy blood and tissue kit (cat no \# 69506) as per manufacturer instructions. Real time PCR was performed on genomic DNA using Fast SYBR green master mix (Applied Biosystems), and results were analyzed with a CFX96 Touch real-time PCR detection system (Bio-Rad). GAPDH was used as an internal control.

\section{Acknowledgments}

We would like to thank J Ron Vale, Stanley Qi, David Root, and S. Diane Hayward for kindly providing plasmids, and Don Ganem, Rolf Renne, Oren Kobiler, Richard F. Ambinder, Bert Vogelstein and Uta Francke for cell lines. We also would like to thank S. Diane Hayward for critically reading the manuscript, and Yosef Shaul for fruitful discussions. We are grateful for the support of the Elias, Genevieve and Georgianna Atol Charitable Trust to the Daniella Lee Casper Laboratory in Viral Oncology. This work was supported by grants from the Israel Science Foundation (https://www.isf.org.il) to M.S. (1134/16), and Research Career Development Award from the Israel Cancer Research Fund (https://www.icrfonline.org/) to M.S. (01282). The funders had no role in study design, data collection and analysis, decision to publish, or preparation of the manuscript.

\section{References}

1. Hall JM, McDonnell DP, Korach KS. Allosteric regulation of estrogen receptor structure, function, and coactivator recruitment by different estrogen response elements. Molecular endocrinology (Baltimore, Md. 2002;16(3):469-86.

2. Kim TK, Maniatis T. The mechanism of transcriptional synergy of an in vitro assembled interferon-beta enhanceosome. Molecular cell. 1997;1(1):119-29.

3. Merika M, Williams AJ, Chen G, Collins T, Thanos D. Recruitment of CBP/p300 by the IFN beta enhanceosome is required for synergistic activation of transcription. Molecular cell. 1998;1(2):277-87.

4. Nan X, Campoy FJ, Bird A. MeCP2 is a transcriptional repressor with abundant binding sites in genomic chromatin. Cell. 1997;88(4):471-81.

5. Jones PL, Veenstra GJ, Wade PA, Vermaak D, Kass SU, Landsberger N, et al. Methylated DNA and $\mathrm{MeCP} 2$ recruit histone deacetylase to repress transcription. Nature genetics. 1998;19(2):187-91.

6. Nan X, Ng HH, Johnson CA, Laherty CD, Turner BM, Eisenman RN, et al. Transcriptional repression by the methyl-CpG-binding protein $\mathrm{MeCP} 2$ involves a histone deacetylase complex. Nature. 1998;393(6683):386-9. 
7. Bannister AJ, Zegerman P, Partridge JF, Miska EA, Thomas JO, Allshire RC, et al. Selective recognition of methylated lysine 9 on histone H3 by the HP1 chromo domain. Nature. 2001;410(6824):120-4.

8. Lachner M, O'Carroll D, Rea S, Mechtler K, Jenuwein T. Methylation of histone H3 lysine 9 creates a binding site for HP1 proteins. Nature. 2001;410(6824):116-20.

9. Agarwal N, Hardt T, Brero A, Nowak D, Rothbauer U, Becker A, et al. MeCP2 interacts with HP1 and modulates its heterochromatin association during myogenic differentiation. Nucleic acids research. 2007;35(16):5402-8.

10. Gonzales ML, Adams S, Dunaway KW, LaSalle JM. Phosphorylation of distinct sites in $\mathrm{MeCP} 2$ modifies cofactor associations and the dynamics of transcriptional regulation. Molecular and cellular biology. 2012;32(14):2894-903.

11. Chang Y, Cesarman E, Pessin MS, Lee F, Culpepper J, Knowles DM, et al. Identification of herpesvirus-like DNA sequences in AIDS-associated Kaposi's sarcoma. Science (New York, NY. 1994;266(5192):1865-9.

12. Henke-Gendo C, Schulz TF. Transmission and disease association of Kaposi's sarcomaassociated herpesvirus: recent developments. Curr Opin Infect Dis. 2004;17(1):53-7.

13. Ye F, Lei X, Gao SJ. Mechanisms of Kaposi's Sarcoma-Associated Herpesvirus Latency and Reactivation. Adv Virol. 2011;2011.

14. Rainbow L, Platt GM, Simpson GR, Sarid R, Gao SJ, Stoiber H, et al. The 222- to 234kilodalton latent nuclear protein (LNA) of Kaposi's sarcoma-associated herpesvirus (human herpesvirus 8) is encoded by orf73 and is a component of the latency-associated nuclear antigen. J Virol. 1997;71(8):5915-21.

15. Kedes DH, Lagunoff M, Renne R, Ganem D. Identification of the gene encoding the major latency-associated nuclear antigen of the Kaposi's sarcoma-associated herpesvirus. J Clin Invest. 1997;100(10):2606-10.

16. Grundhoff A, Ganem D. The latency-associated nuclear antigen of Kaposi's sarcomaassociated herpesvirus permits replication of terminal repeat-containing plasmids. J Virol. 2003;77(4):2779-83.

17. $\mathrm{Hu}$ J, Garber AC, Renne R. The latency-associated nuclear antigen of Kaposi's sarcomaassociated herpesvirus supports latent DNA replication in dividing cells. J Virol. 2002;76(22):11677-87.

18. Kelley-Clarke B, De Leon-Vazquez E, Slain K, Barbera AJ, Kaye KM. Role of Kaposi's sarcoma-associated herpesvirus C-terminal LANA chromosome binding in episome persistence. J Virol. 2009;83(9):4326-37.

19. De Leon Vazquez E, Kaye KM. The internal Kaposi's sarcoma-associated herpesvirus LANA regions exert a critical role on episome persistence. J Virol. 2011;85(15):7622-33.

20. Hellert J, Weidner-Glunde M, Krausze J, Lünsdorf H, Ritter C, Schulz TF, et al. The 3D structure of Kaposi sarcoma herpesvirus LANA C-terminal domain bound to DNA. Proceedings of the National Academy of Sciences of the United States of America. 2015;112(21):6694-9.

21. Garber AC, Hu J, Renne R. Latency-associated nuclear antigen (LANA) cooperatively binds to two sites within the terminal repeat, and both sites contribute to the ability of LANA to suppress transcription and to facilitate DNA replication. J Biol Chem. 2002;277(30):27401-11.

22. Domsic JF, Chen HS, Lu F, Marmorstein R, Lieberman PM. Molecular basis for oligomeric-DNA binding and episome maintenance by KSHV LANA. PLoS Pathog. 2013;9(10):e1003672. 
23. De Leo A, Deng Z, Vladimirova O, Chen HS, Dheekollu J, Calderon A, et al. LANA oligomeric architecture is essential for $\mathrm{KSHV}$ nuclear body formation and viral genome maintenance during latency. PLoS Pathog. 2019;15(1):e1007489.

24. Jinek M, Chylinski K, Fonfara I, Hauer M, Doudna JA, Charpentier E. A programmable dual-RNA-guided DNA endonuclease in adaptive bacterial immunity. Science (New York, NY. 2012;337(6096):816-21.

25. Qi LS, Larson MH, Gilbert LA, Doudna JA, Weissman JS, Arkin AP, et al. Repurposing CRISPR as an RNA-guided platform for sequence-specific control of gene expression. Cell. 2013;152(5):1173-83.

26. Gilbert LA, Larson MH, Morsut L, Liu Z, Brar GA, Torres SE, et al. CRISPR-mediated modular RNA-guided regulation of transcription in eukaryotes. Cell. 2013;154(2):442-51.

27. Deng W, Shi X, Tjian R, Lionnet T, Singer RH. CASFISH: CRISPR/Cas9-mediated in situ labeling of genomic loci in fixed cells. Proceedings of the National Academy of Sciences of the United States of America. 2015;112(38):11870-5.

28. Chen B, Gilbert LA, Cimini BA, Schnitzbauer J, Zhang W, Li GW, et al. Dynamic imaging of genomic loci in living human cells by an optimized CRISPR/Cas system. Cell. 2013;155(7):1479-91.

29. Anton T, Bultmann S, Leonhardt H, Markaki Y. Visualization of specific DNA sequences in living mouse embryonic stem cells with a programmable fluorescent CRISPR/Cas system. Nucleus. 2014;5(2):163-72.

30. Duan J, Lu G, Hong Y, Hu Q, Mai X, Guo J, et al. Live imaging and tracking of genome regions in CRISPR/dCas9 knock-in mice. Genome Biol. 2018;19(1):192.

31. Tanenbaum ME, Gilbert LA, Qi LS, Weissman JS, Vale RD. A protein-tagging system for signal amplification in gene expression and fluorescence imaging. Cell. 2014;159(3):635-46.

32. Ballestas ME, Chatis PA, Kaye KM. Efficient persistence of extrachromosomal KSHV DNA mediated by latency-associated nuclear antigen. Science (New York, NY. 1999;284(5414):641-4.

33. Kelley-Clarke B, Ballestas ME, Srinivasan V, Barbera AJ, Komatsu T, Harris TA, et al. Determination of Kaposi's sarcoma-associated herpesvirus C-terminal latency-associated nuclear antigen residues mediating chromosome association and DNA binding. J Virol. 2007;81(8):434856.

34. Hellert J, Weidner-Glunde M, Krausze J, Richter U, Adler H, Fedorov R, et al. A structural basis for BRD2/4-mediated host chromatin interaction and oligomer assembly of Kaposi sarcomaassociated herpesvirus and murine gammaherpesvirus LANA proteins. PLoS Pathog. 2013;9(10):e1003640.

35. Chiu YF, Sugden AU, Fox K, Hayes M, Sugden B. Kaposi's sarcoma-associated herpesvirus stably clusters its genomes across generations to maintain itself extrachromosomally. The Journal of cell biology. 2017;216(9):2745-58.

36. Stedman W, Deng Z, Lu F, Lieberman PM. ORC, MCM, and Histone Hyperacetylation at the Kaposi's Sarcoma-Associated Herpesvirus Latent Replication Origin. J Virol. 2004;78(22):12566-75.

37. Verma SC, Lan K, Choudhuri T, Cotter MA, Robertson ES. An autonomous replicating element within the KSHV genome. Cell host \& microbe. 2007;2(2):106-18. 
38. Krithivas A, Young DB, Liao G, Greene D, Hayward SD. Human herpesvirus 8 LANA interacts with proteins of the $\mathrm{mSin} 3$ corepressor complex and negatively regulates Epstein-Barr virus gene expression in dually infected PEL cells. J Virol. 2000;74(20):9637-45.

39. Cai Q, Cai S, Zhu C, Verma SC, Choi JY, Robertson ES. A unique SUMO-2-interacting motif within LANA is essential for KSHV latency. PLoS Pathog. 2013;9(11):e1003750.

40. Krithivas A, Fujimuro M, Weidner M, Young DB, Hayward SD. Protein interactions targeting the latency-associated nuclear antigen of Kaposi's sarcoma-associated herpesvirus to cell chromosomes. J Virol. 2002;76(22):11596-604.

41. Matsumura S, Persson LM, Wong L, Wilson AC. The latency-associated nuclear antigen interacts with MeCP2 and nucleosomes through separate domains. J Virol. 2010;84(5):2318-30. 42. Verma SC, Cai Q, Kreider E, Lu J, Robertson ES. Comprehensive analysis of LANA interacting proteins essential for viral genome tethering and persistence. PloS one. 2013;8(9):e74662.

43. Stuber G, Mattsson K, Flaberg E, Kati E, Markasz L, Sheldon JA, et al. HHV-8 encoded LANA-1 alters the higher organization of the cell nucleus. Molecular cancer. 2007;6:28.

44. Rhee I, Bachman KE, Park BH, Jair KW, Yen RW, Schuebel KE, et al. DNMT1 and DNMT3b cooperate to silence genes in human cancer cells. Nature. 2002;416(6880):552-6.

45. Barbera AJ, Chodaparambil JV, Kelley-Clarke B, Joukov V, Walter JC, Luger K, et al. The Nucleosomal Surface as a Docking Station for Kaposi's Sarcoma Herpesvirus LANA. Science (New York, NY. 2006;311(5762):856-61.

46. Viejo-Borbolla A, Ottinger M, Bruning E, Burger A, Konig R, Kati E, et al. Brd2/RING3 interacts with a chromatin-binding domain in the Kaposi's Sarcoma-associated herpesvirus latency-associated nuclear antigen 1 (LANA-1) that is required for multiple functions of LANA1. J Virol. 2005;79(21):13618-29.

47. Ghosh RP, Horowitz-Scherer RA, Nikitina T, Gierasch LM, Woodcock CL. Rett syndrome-causing mutations in human $\mathrm{MeCP} 2$ result in diverse structural changes that impact folding and DNA interactions. J Biol Chem. 2008;283(29):20523-34.

48. Traynor J, Agarwal P, Lazzeroni L, Francke U. Gene expression patterns vary in clonal cell cultures from Rett syndrome females with eight different MECP2 mutations. BMC Med Genet. 2002;3:12.

49. Griffiths R, Whitehouse A. Herpesvirus saimiri episomal persistence is maintained via interaction between open reading frame 73 and the cellular chromosome-associated protein MeCP2. J Virol. 2007;81(8):4021-32.

50. Dedon PC, Soults JA, Allis CD, Gorovsky MA. A simplified formaldehyde fixation and immunoprecipitation technique for studying protein-DNA interactions. Anal Biochem. 1991;197(1):83-90.

51. Orlando V, Paro R. Mapping Polycomb-repressed domains in the bithorax complex using in vivo formaldehyde cross-linked chromatin. Cell. 1993;75(6):1187-98.

52. Aronheim A, Zandi E, Hennemann H, Elledge SJ, Karin M. Isolation of an AP-1 repressor by a novel method for detecting protein-protein interactions. Molecular and cellular biology. 1997;17(6):3094-102.

53. Gonzalez-Serricchio AS, Sternberg PW. Visualization of C. elegans transgenic arrays by GFP. BMC Genet. 2006;7:36. 


\section{1}

54. Higa M, Kushiyama T, Kurashige S, Kohmon D, Enokitani K, Iwahori S, et al. TRF2 recruits ORC through TRFH domain dimerization. Biochim Biophys Acta Mol Cell Res. 2017;1864(1):191-201.

55. Gao Y, Han M, Shang S, Wang H, Qi LS. Interrogation of the dynamic properties of higherorder heterochromatin using CRISPR-dCas9. Molecular cell. 2021.

56. Shin Y, Chang YC, Lee DSW, Berry J, Sanders DW, Ronceray P, et al. Liquid Nuclear Condensates Mechanically Sense and Restructure the Genome. Cell. 2018;175(6):1481-91 e13. 57. Vladimirova O, De Leo A, Deng Z, Wiedmer A, Hayden J, Lieberman PM. Phase separation and DAXX redistribution contribute to LANA nuclear body and KSHV genome dynamics during latency and reactivation. PLoS Pathog. 2021;17(1):e1009231.

58. Wang L, Hu M, Zuo MQ, Zhao J, Wu D, Huang L, et al. Rett syndrome-causing mutations compromise MeCP2-mediated liquid-liquid phase separation of chromatin. Cell Res. 2020;30(5):393-407.

59. Calderwood M, White RE, Griffiths RA, Whitehouse A. Open reading frame 73 is required for herpesvirus saimiri A11-S4 episomal persistence. J Gen Virol. 2005;86(Pt 10):2703-8.

60. Adams VH, McBryant SJ, Wade PA, Woodcock CL, Hansen JC. Intrinsic disorder and autonomous domain function in the multifunctional nuclear protein, MeCP2. J Biol Chem. 2007;282(20):15057-64.

61. Ghosh RP, Horowitz-Scherer RA, Nikitina T, Shlyakhtenko LS, Woodcock CL. MeCP2 binds cooperatively to its substrate and competes with histone $\mathrm{H} 1$ for chromatin binding sites. Molecular and cellular biology. 2010;30(19):4656-70.

62. Fujimuro M, Hayward SD. The latency-associated nuclear antigen of Kaposi's sarcomaassociated herpesvirus manipulates the activity of glycogen synthase kinase-3beta. J Virol. 2003;77(14):8019-30.

63. Myoung J, Ganem D. Infection of lymphoblastoid cell lines by Kaposi's sarcomaassociated herpesvirus: critical role of cell-associated virus. J Virol. 2011;85(19):9767-77.

2

3

.

595

596

97

8

9

00


601

602

603

604

605

606

607

608

609

610

611

612

613

614

615

616

617

618

619

620

621

622

623

624

625

626

627

628

629

630

\section{Figure legends}

Figure 1. Schematic illustration of CRISPR-PITA. (A) Schematic illustration of dCas9-SunTag, sgRNA and Fc-LANA or other protein of interest (Fc-pY), and their binding to repetitive elements. (B) SLK cells were transfected with dCas9-SunTag, Fc-LANA, and sgTelomere as illustrated on the left of the images. Immunofluorescence assay was performed to detect LANA (red), and the nucleus was stained with DAPI (blue). Scale bar $=5 \mu \mathrm{m}$. (C) Schematic illustration of the CRISPR-PITA, targeting of Fc-LANA or any other protein of interest to the telomeres via dCas9. Then the recruitment of other nuclear proteins to these dots is evaluated via immunostaining.

Figure 2. LANA recruits ORC2 and SIN3A but not MeCP2. SLK cells were transfected with dCas9SunTag, Fc-LANA, and sgTelomere expression plasmids as can be seen in the illustrations on the left. Immunofluorescence assays were performed to detect LANA (red) and ORC2 (A) mSin3a (B) or MeCP2 (C) (in green) cellular localization. The nucleus was stained with DAPI. Scale bar $=5 \mu \mathrm{m}$. The average ratio between big and small foci for each analysed protein is presented on the right, based on the threshold $\geq 0.15$ micron $^{2},(\mathrm{n}=15)$. One-tailed T-tests were performed on the ratios $(*, \mathrm{P} \leq 0.05 ; * *, \mathrm{P} \leq 0.01, * * *, \mathrm{P} \leq$ $0.001)$. Results are presented as mean $+/-$ SD.

Figure 3. MeCP2 recruits LANA. SLK cells were transfected with dCas9-SunTag, Fc-MeCP2, and sgTelomere in combination with GFP-LANA (A) or GFP (B) as illustrated on the left. Immunofluorescence assay was performed to detect Fc-MeCP2, or fluorescently labelled GFP-LANA and GFP. The nucleus was stained with DAPI. Scale bar $=5 \mu \mathrm{m}$. The average ratio between big and small foci are presented on the right, same as in Fig 2. (C) SLK cells were transfected with FLAG-LANA N+C, FLAG-LANA C, or FLAG-LANA N, same as in A. Immunofluorescence assay was performed to detect both Fc-MeCP2 and LANA.

Figure 4. MeCP2 recruits HDAC1, while HDAC1 cannot recruit MeCP2. (A) HCT cells were transfected with dCas9-SunTag, Fc-MeCP2 and sgTelomere as illustrated on the left. Immunofluorescence assay was performed to detect HDAC1 (green) and Fc-MeCP2 (red). (B-C) HCT (B) and HCT DKO (C) cells were transfected same as in A but with Fc-HDAC1. Immunofluorescence assay was performed to detect MeCP2 (green) and Fc-HDAC1 (red). The nucleus was stained with DAPI. Scale bar $=5 \mu \mathrm{m}$. The average ratio between big and small foci are presented on the right, same as in Fig 2.

Figure 5. HP1 $\alpha$ recruits MeCP2. SLK cells were transfected with dCas9-SunTag, Fc-HP1 $\alpha$, and sgTelomere as illustrated on the left. Immunofluorescence assay was performed to detect MeCP2 (green) 

and small foci are presented on the right, same as in Fig 2.

633

634

635

636

637

638

639

640

641

642

643

644

645

646

647

648

649

650

651

652

653

654

655

656

Figure 6. Rett syndrome mutant MeCP2 T158M cannot support KSHV latency. (A-B) LCLs were infected with rKSHV.219 and GFP-positive cells by FACS analysis (A) or amount of KSHV DNA by qPCR (B) were evaluated at the indicated time points. Experiment was performed with biological triplicates. (C) Immunofluorescence assay was performed to detect GFP-LANA (green) and Fc- MeCP2 (red). The nucleus was stained with DAPI. Scale bar $=5 \mu \mathrm{m}$. The average ratio between big and small foci are presented on the right, same as in Fig 2. (D) NIH 3 T3 cells were transfected with GFP-LANA and wt MeCP2, T158M mutant, or del-MBD. Immunofluorescence assay was performed same as in C. Intensity profiles along the indicated arrows are presented on the right.

Figure 7. Schematic presentation of directional recruitment. (A) In its DNA free form, the MBD and TRD of MeCP2 are not available for LANA interaction. (B) Upon binding to methylated DNA LANA binding surface in $\mathrm{MeCP} 2$ becomes available for LANA binding. (C) LANA tethers the viral episomal genomes to cellular chromosomes by binding to histones via its $\mathrm{N}$-terminal domain and to MeCP2 via its C-terminal domain. This unidirectional recruitment of LANA by MeCP2 is essential for viral genome maintenance during latency.

(1)

(1)

\section{9}

\section{0}


FIG S1. LANA-telomere dots co-localize with the telomeric protein TRF2. SLK cells were transfected with dCas9-SunTag, Fc-LANA (A \& B) or Fc-LANA oligomerization mutant (B), and sgTelomere as

660 illustrated on the left of the images. Immunofluorescence assay was performed to detect LANA (red) and 661 TRF2 (white), and the nucleus was stained with DAPI. Scale bar $=5 \mu \mathrm{m}$.

FIG S2. Fc-LANA interacts with MeCP2. HEK 293T cells were co-transfected with Fc-LANA (FcFLAG-LANA) and MeCP2 expression vectors. $48 \mathrm{~h}$ after transfection cells were lysed in lysis buffer

664 (50mM Tris- $\mathrm{HCl} \mathrm{pH} 7.9 ; 100 \mathrm{mM} \mathrm{NaCl} ; 0.5 \mathrm{mM}$ EDTA; $\%$ Glycerol; $0.2 \%$ NP40 and Protease 665 inhibitor cocktail (Halt ${ }^{\mathrm{TM}}$ Protease Inhibitor Cocktail (100X) Thermo Scientific ${ }^{\mathrm{TM}}$ ). Cell extracts were 666 immunoprecipitated with anti-FLAG beads and subjected to SDS-PAGE and Western blot analysis with 667 anti-MeCP2 (upper and lower panels), or anti-FLAG (middle panel) antibodies.

FIG S3. LANA cannot recruit MeCP2 even in HCT-DKO cells. HCT116 (A) and DKO (DNMT3B -/and DNMT1 -/-) HCT116 cells (B) were transfected with dCas9-SunTag, Fc-LANA, and sgTelomere as

670 illustrated on the left of the images. Immunofluorescence assay was performed to detect MeCP2 (green)

671 and LANA (red). The nucleus was stained with DAPI. Scale bar $=5 \mu \mathrm{m}$.

672 FIG S4. ORC2 and SIN3A but not MeCP2 co-localize with LANA dots in KSHV infected cells.

673 Immunofluorescence assays were performed to detect ORC2 (A) SIN3A (B) or MeCP2 (C) and LANA 674 (red) cellular localization in KSHV-negative (BJAB) and KSHV-positive (BCBL1) lymphoma cell lines. 675 The nucleus was stained with DAPI. Scale bar $=5 \mu \mathrm{m}$.

\section{Table S1. Primers used in this study.}


bioRxiv preprint doi: https://doi.org/10.1101/2021.10.10.463816; this version posted October 10, 2021. The copyright holder for this preprint A (which was not certified by peer review) is the author/funder. All rights reserved. No reuse allowed without permission.
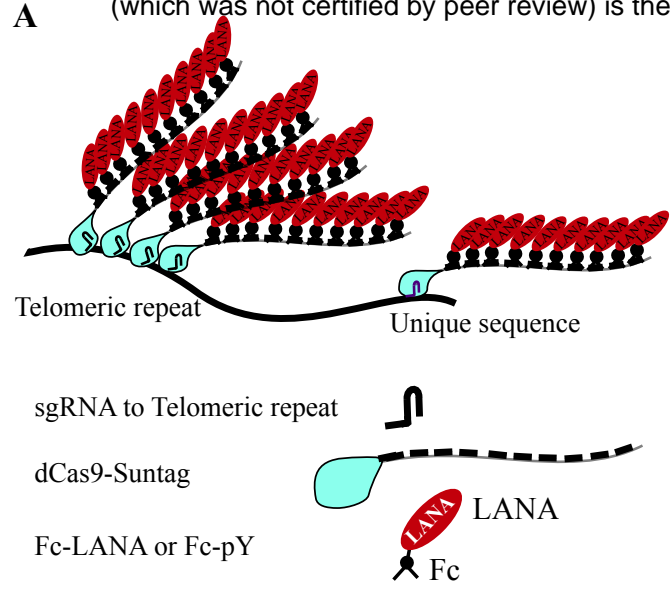

B

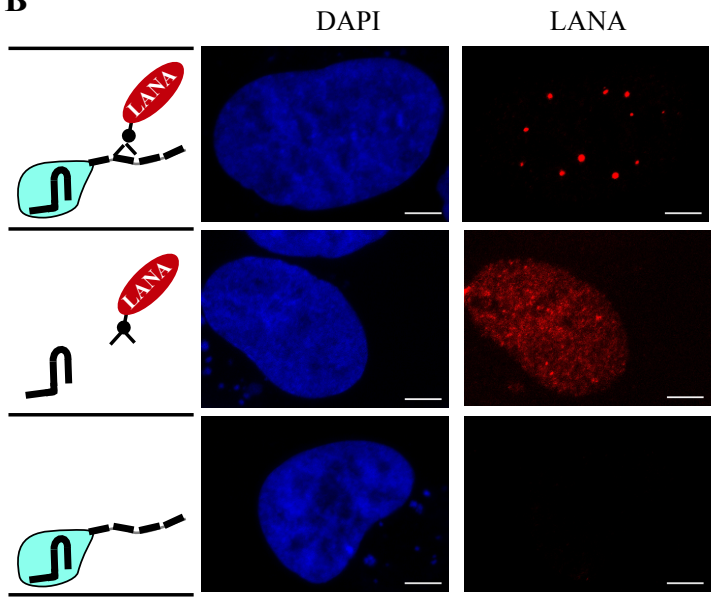

C

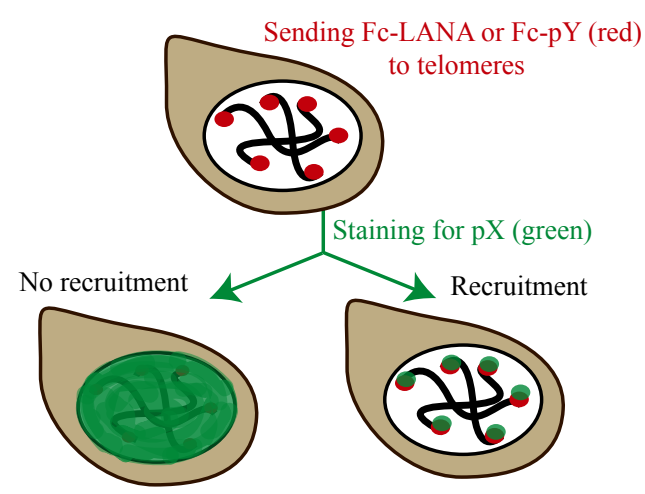

Figure 1. Schematic illustration of CRISPR-PITA. (A) Schematic illustration of dCas9-SunTag, sgRNA and Fc-LANA or other protein of interest (Fc-pY), and their binding to repetitive elements. (B) SLK cells were transfected with dCas9-SunTag, Fc-LANA, and sgTelomere as illustrated on the left of the images. Immunofluorescence assay was performed to detect LANA (red), and the nucleus was stained with DAPI (blue). Scale bar $=5 \mu \mathrm{m}$. (C) Schematic illustration of the CRISPR-PITA, targeting of Fc-LANA or any other protein of interest to the telomeres via dCas9. Then the recruitment of other nuclear proteins to these dots is evaluated via immunostaining. 
bioRxiv preprint doi: https://doi.org/10.1101/2021.10.10.463816; this version posted October 10, 2021. The copyright holder for this preprint (which was not certified by peer review) is the author/funder. All rights reserved. No reuse allowed without permission.

A

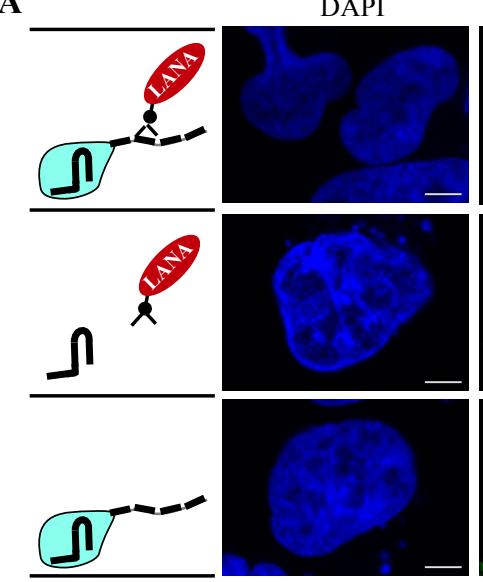

B

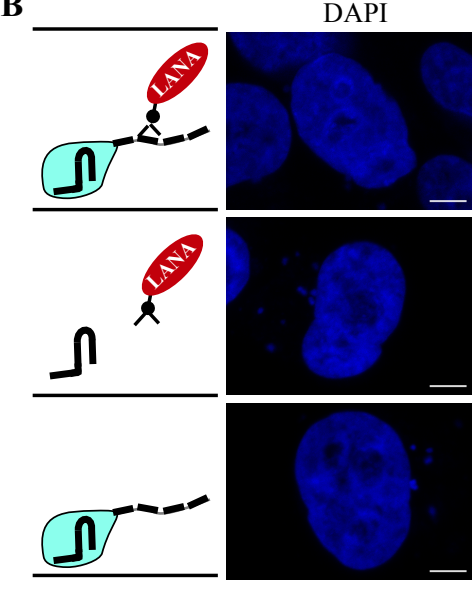

C

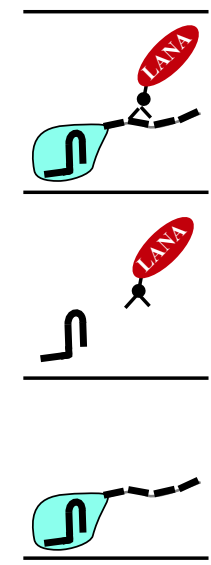

ORC2

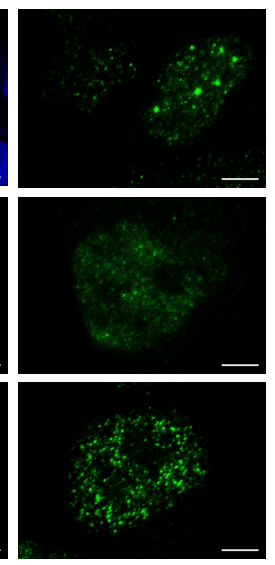

SIN3A

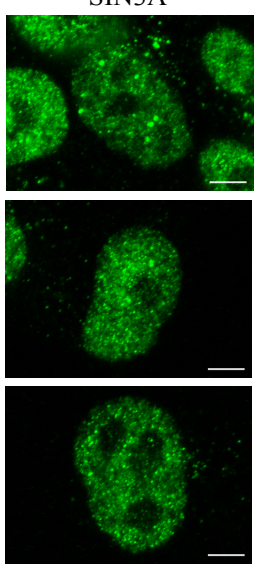

$\mathrm{MeCP} 2$

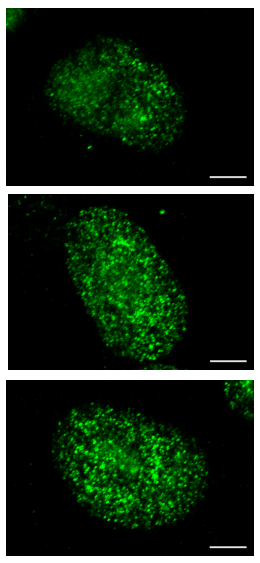

Fc-LANA

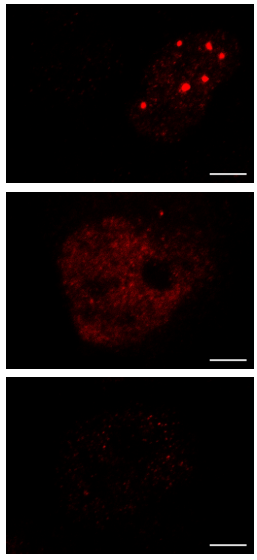

Fc-LANA

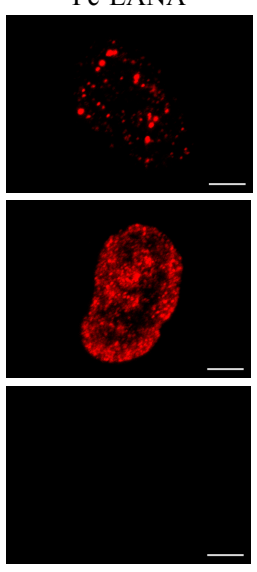

Fc-LANA

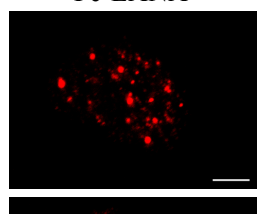

merge

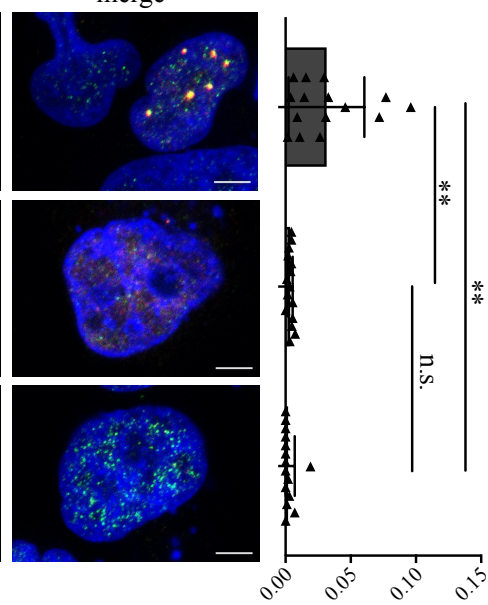

merge
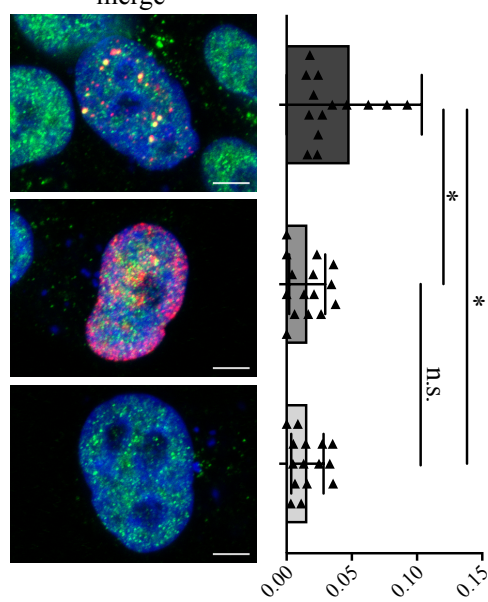

merge
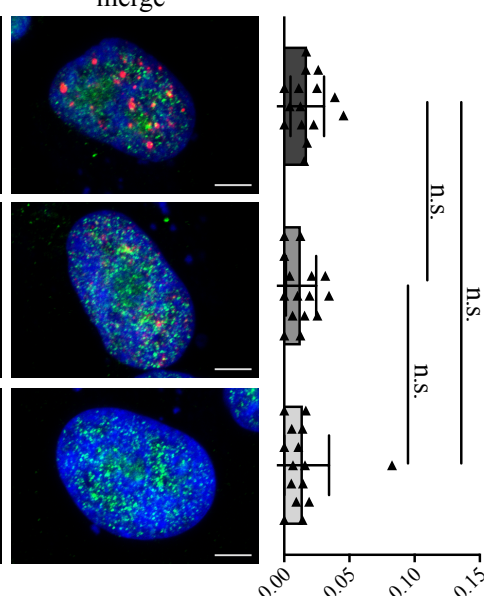

average (large/small dots)

Figure 2. LANA recruits ORC2 and SIN3A but not MeCP2. SLK cells were transfected with dCas9SunTag, Fc-LANA, and sgTelomere expression plasmids as can be seen in the illustrations on the left. Immunofluorescence assays were performed to detect LANA (red) and ORC2 (A) mSinza (B) or MeCP2 (C) (in green) cellular localization. The nucleus was stained with DAPI. Scale bar $=5 \mu \mathrm{m}$. The average ratio between big and small foci for each analysed protein is presented on the right, based on the threshold $\geq 0.15$ micron $^{2},(\mathrm{n}=15)$. One-tailed T-tests were performed on the ratios $\left({ }^{*}, \mathrm{P} \leq 0.05 ;{ }^{* *}, \mathrm{P} \leq 0.01,{ }^{* * *}, \mathrm{P} \leq 0.001\right)$. Results are presented as mean $+/-$ SD. 
A

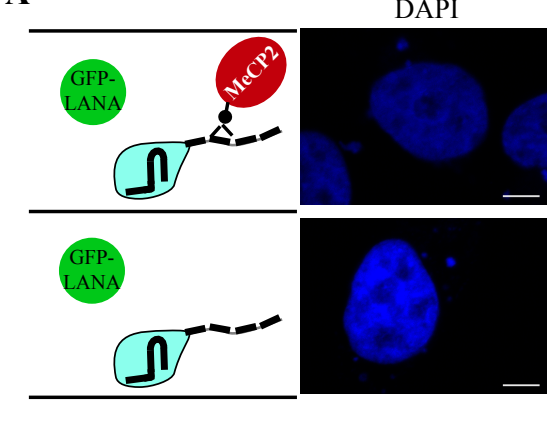

B

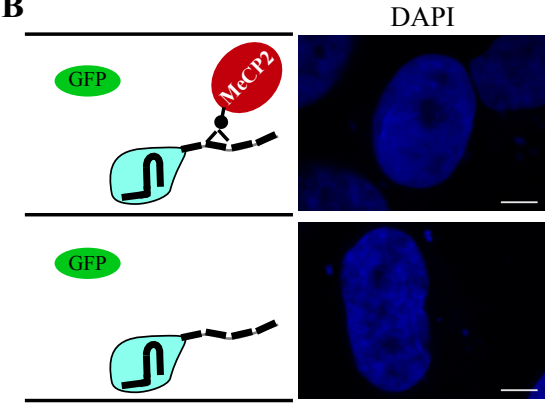

C

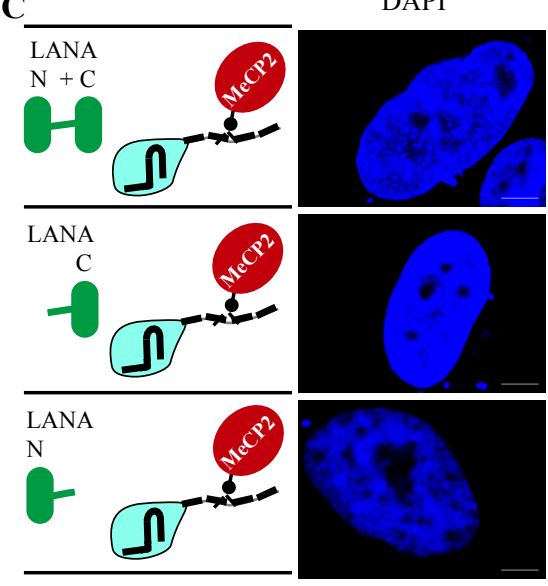

GFP-LANA
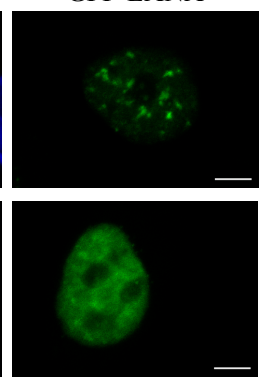

GFP
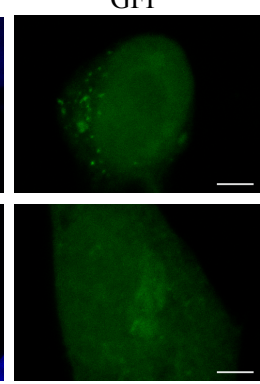

LANA

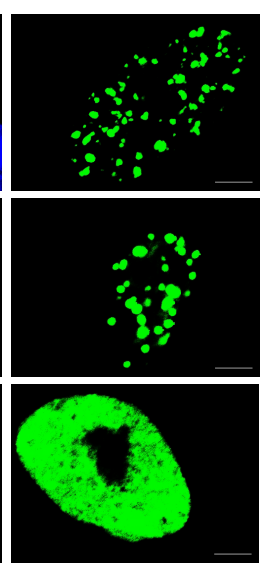

Fc-MeCP2

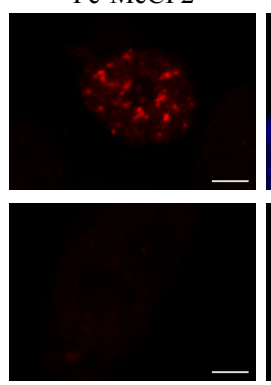

$\mathrm{Fc}-\mathrm{MeCP} 2$
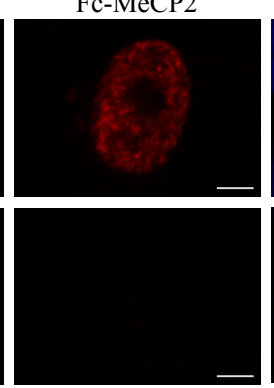

$\mathrm{Fc}-\mathrm{MeCP} 2$

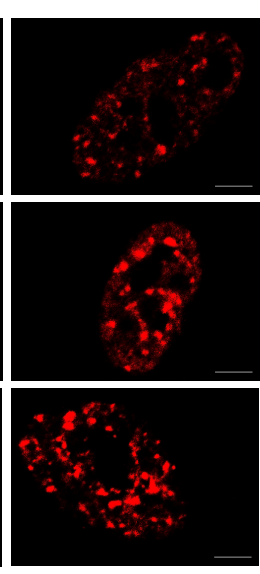

merge
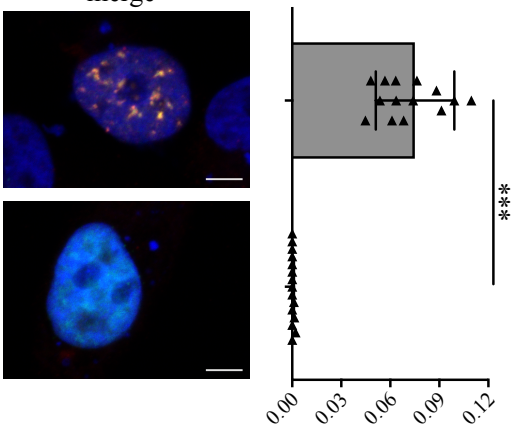

merge
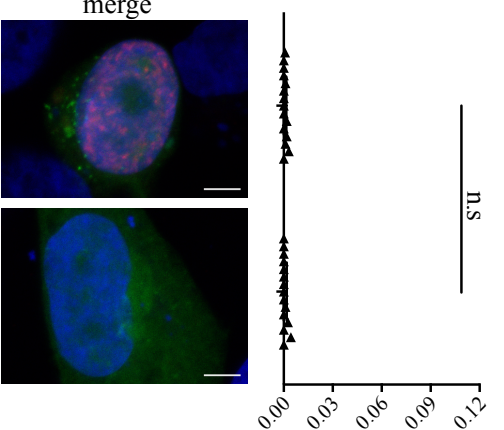

merge
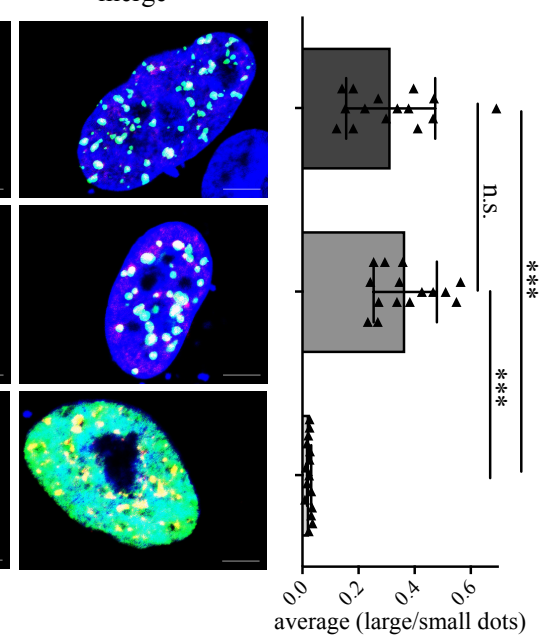

Figure 3. MeCP2 recruits LANA. SLK cells were transfected with dCas9-SunTag, Fc-MeCP2, and sgTelomere in combination with GFP-LANA (A) or GFP (B) as illustrated on the left. Immunofluorescence assay was performed to detect Fc-MeCP2, or fluorescently labelled GFP-LANA and GFP. The nucleus was stained with DAPI. Scale bar $=5 \mu \mathrm{m}$. The average ratio between big and small foci are presented on the right, same as in Fig 2. (C) SLK cells were transfected with FLAG-LANA N+C, FLAG-LANA C, or FLAGLANA N, same as in A. Immunofluorescence assay was performed to detect both Fc-MeCP2 and LANA. 
bioRxiv preprint doi: https://doi.org/10.1101/2021.10.10.463816; this version posted October 10, 2021. The copyright holder for this preprint (which was not certified by peer review) is the author/funder. All rights reserved. No reuse allowed without permission.

A

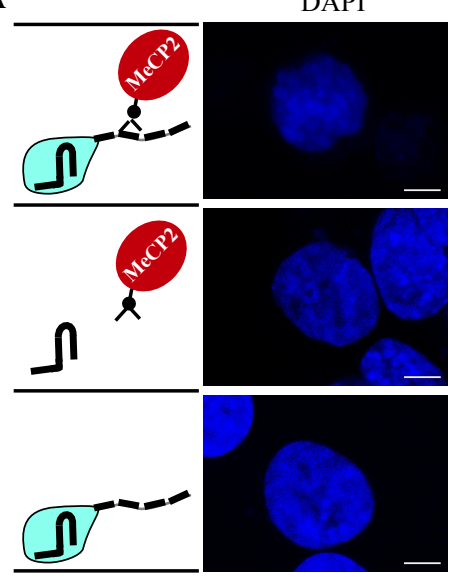

B

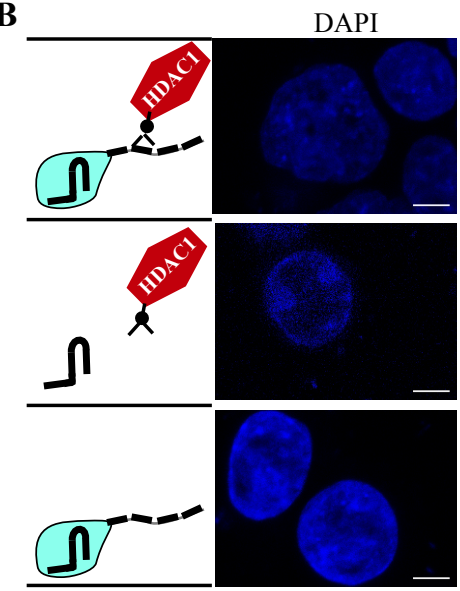

$\mathbf{C}$
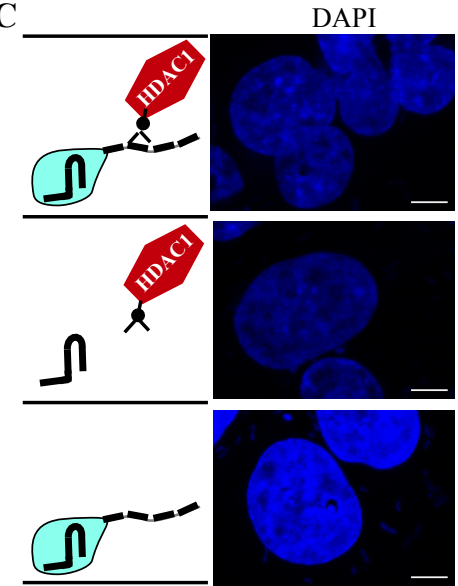

HDAC1

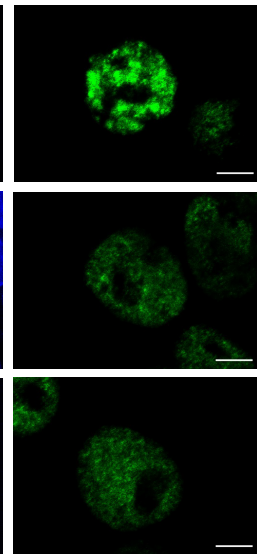

$\mathrm{MeCP} 2$
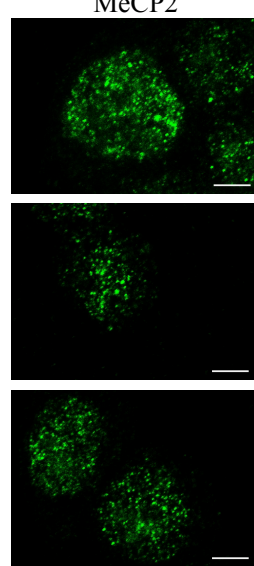

$\mathrm{MeCP} 2$
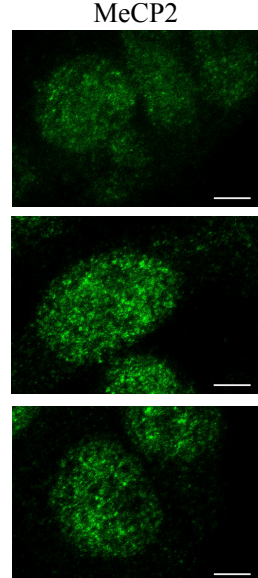

Fc-MeCP2

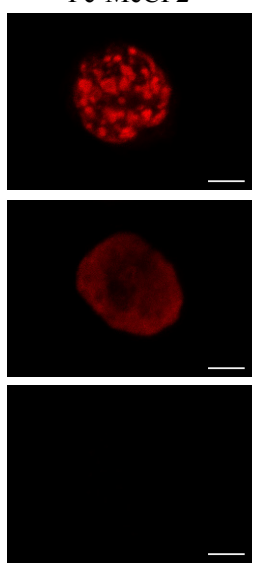

Fc-HDAC

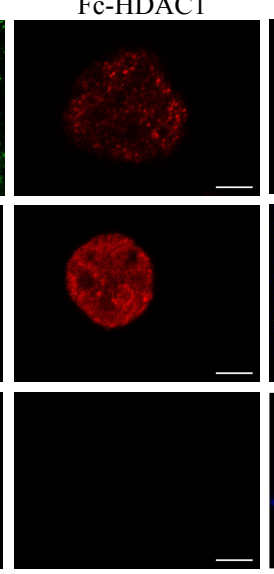

Fc-HDAC1

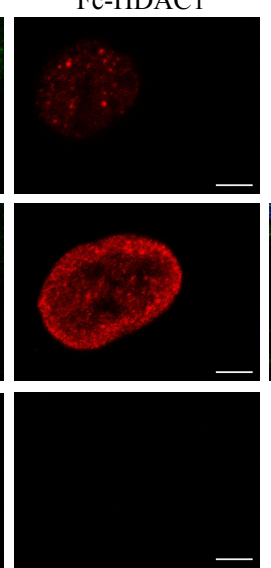

merge
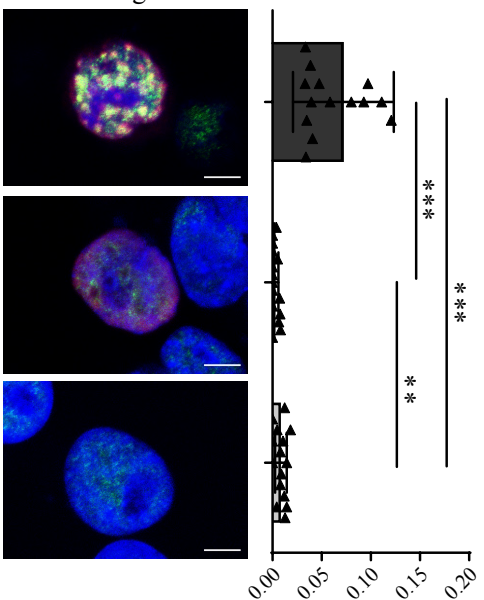

merge
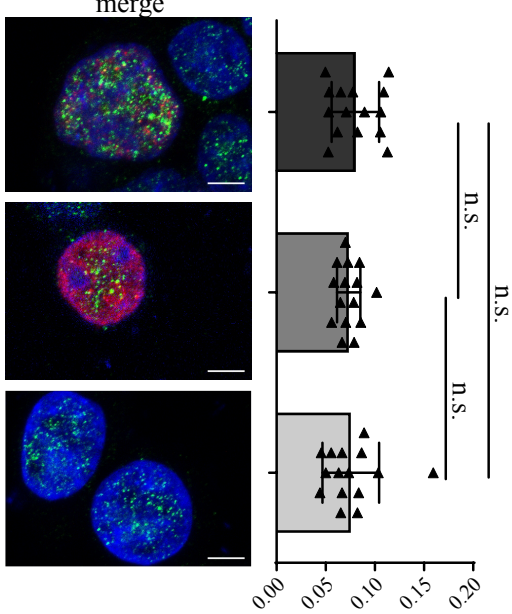

merge

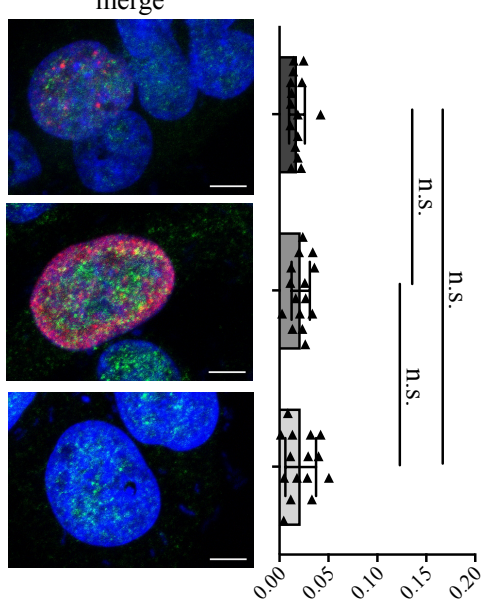

average (large/small dots)

Figure 4. MeCP2 recruits HDAC1, while HDAC1 cannot recruit MeCP2. (A) HCT cells were transfected with dCas9-SunTag, Fc-MeCP2 and sgTelomere as illustrated on the left. Immunofluorescence assay was performed to detect HDAC1 (green) and Fc-MeCP2 (red). (B-C) HCT (B) and HCT DKO (C) cells were transfected same as in A but with Fc-HDAC1. Immunofluorescence assay was performed to detect $\mathrm{MeCP} 2$ (green) and Fc-HDAC1 (red). The nucleus was stained with DAPI. Scale bar $=5 \mu \mathrm{m}$. The average ratio between big and small foci are presented on the right, same as in Fig 2. 
bioRxiv preprint doi: https://doi.org/10.1101/2021.10.10.463816; this version posted October 10, 2021. The copyright holder for this preprint (which was not certified by peer review) is the author/funder. All rights reserved. No reuse allowed without permission.

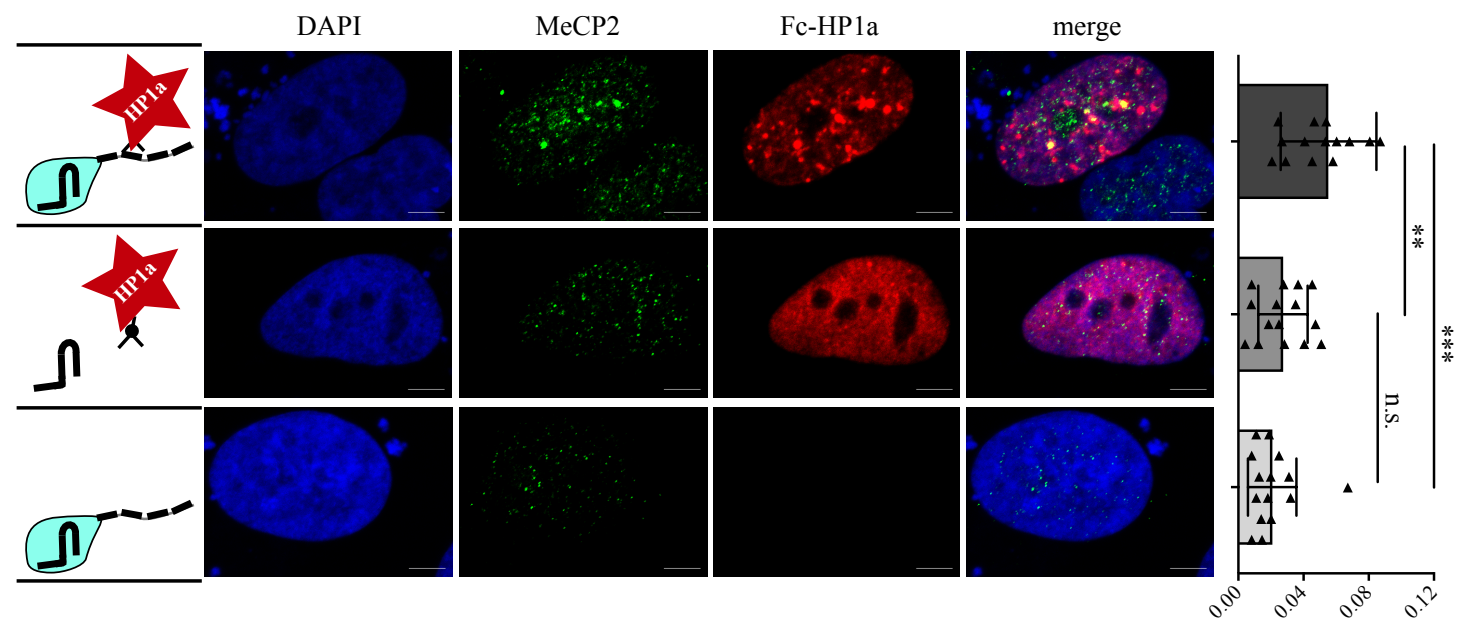

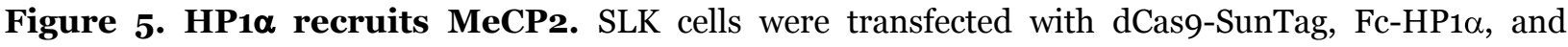
sgTelomere as illustrated on the left. Immunofluorescence assay was performed to detect MeCP2 (green) and Fc-HP1 $\alpha$ (red). The nucleus was stained with DAPI. Scale bar $=5 \mu \mathrm{m}$. The average ratio between big and small foci are presented on the right, same as in Fig 2. 
bioRxiv preprint doi: https://doi.org/10.1101/2021.10.10.463816; this version posted October 10, 2021. The copyright holder for this preprint (which was not certified by peer review) is the author/funder. All rights reserved. No reuse allowed without permission.

A

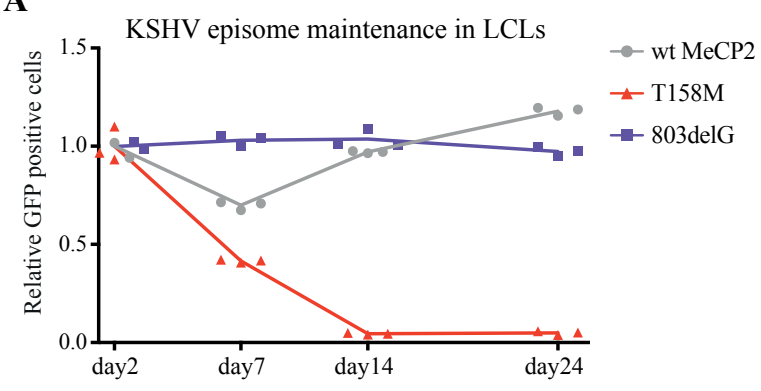

B

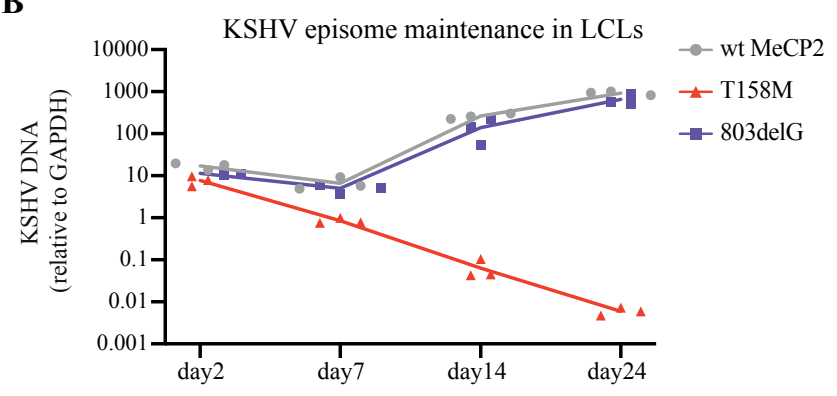

C

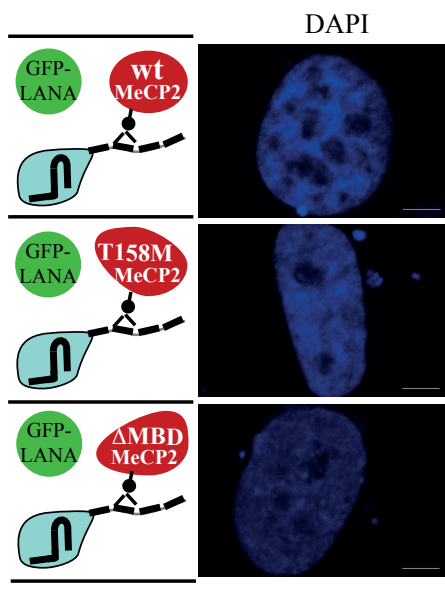

D

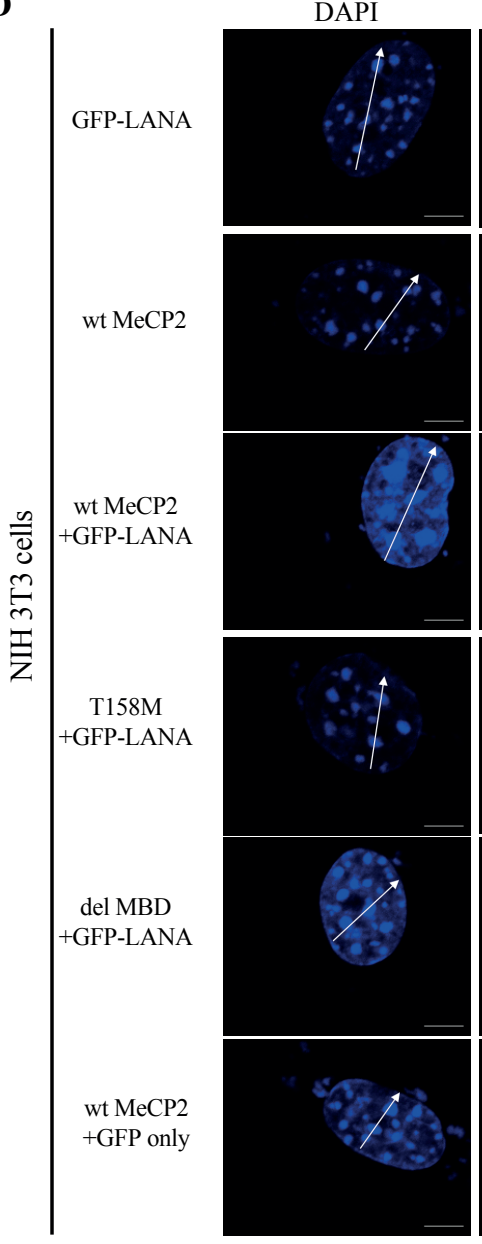

LANA

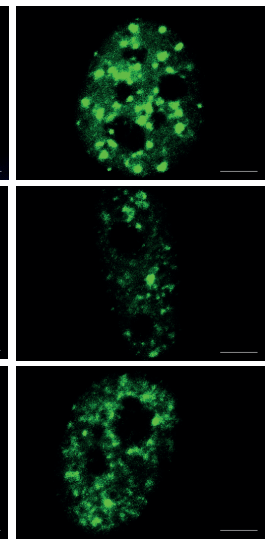

$\mathrm{Fc}-\mathrm{MeCP} 2$

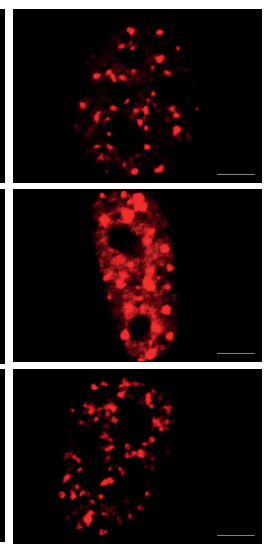

merge
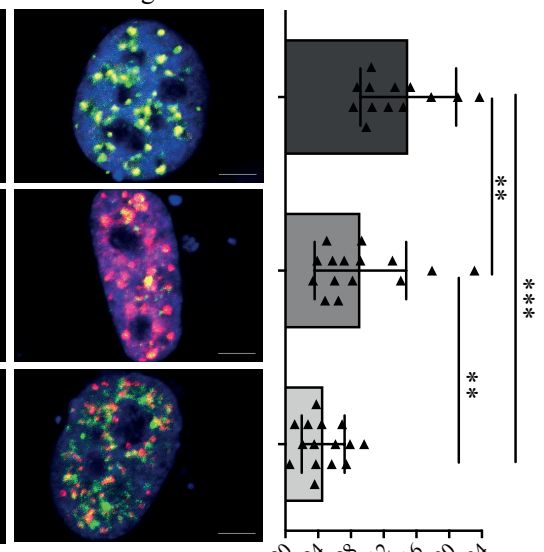

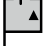

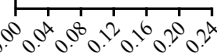

average (large/small dots)
GFP
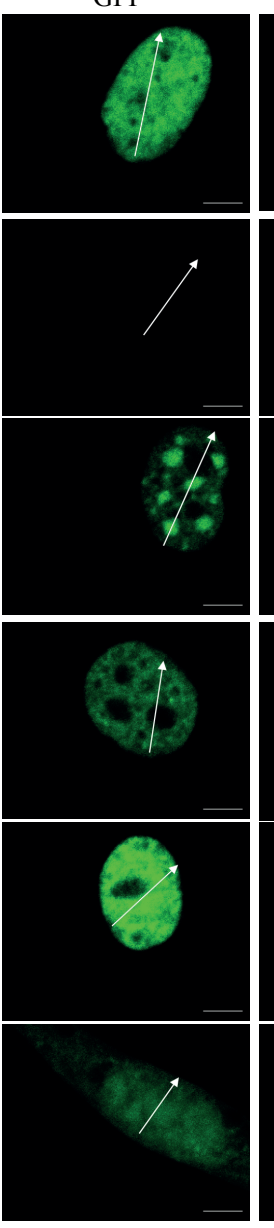

$\mathrm{MeCP} 2$
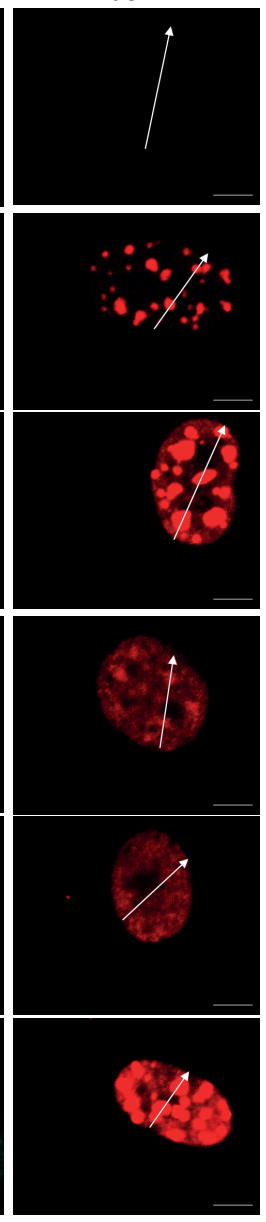

merge
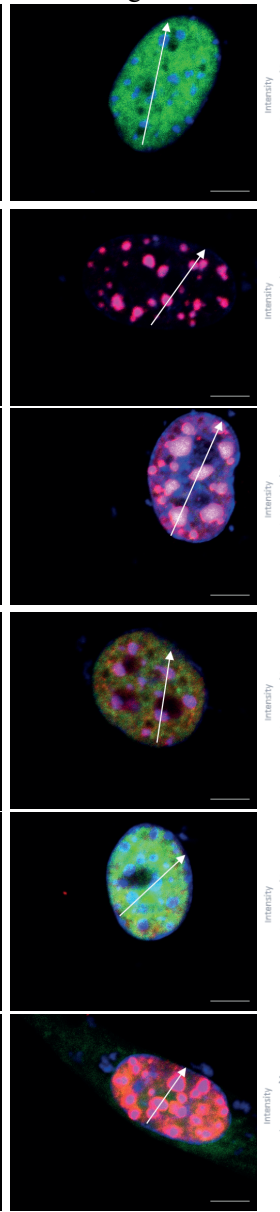
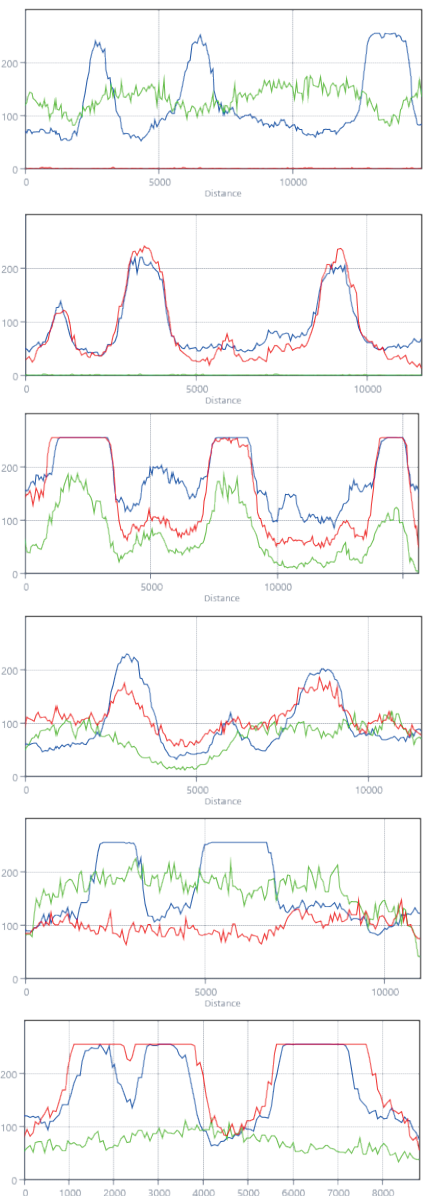


\section{Legend to Figure 6 on previous page}

Figure 6. Rett syndrome mutant MeCP2 T158M cannot support KSHV latency. (A-B) LCLs were infected with rKSHV.219 and GFP-positive cells by FACS analysis (A) or amount of KSHV DNA by qPCR (B) were evaluated at the indicated time points. Experiment was performed with biological triplicates. (C) Immunofluorescence assay was performed to detect GFP-LANA (green) and Fc-MeCP2 (red). The nucleus was stained with DAPI. Scale bar $=5 \mu \mathrm{m}$. The average ratio between big and small foci are presented on the right, same as in Fig 2. (D) NIH $3 \mathrm{~T}_{3}$ cells were transfected with GFP-LANA and wt MeCP2, T158M mutant, or del-MBD. Immunofluorescence assay was performed same as in C. Intensity profiles along the indicated arrows are presented on the right.

A

$\frac{\text { Free MeCP2 }}{\text { LANA binding surface }}$

is not available

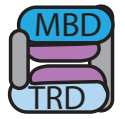

$\mathrm{MeCP2}$
B

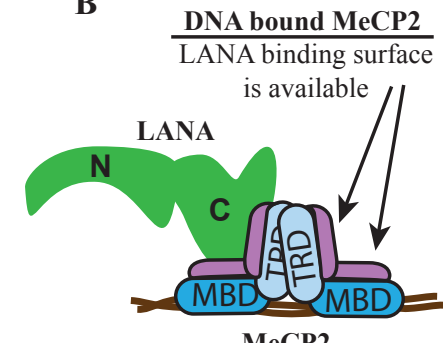

MeCP2

C

LANA binding to both histones and DNA bound MeCP2 enables

genome maintenance

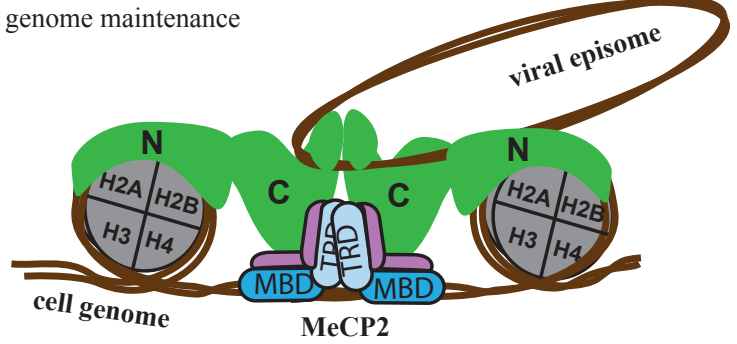

Figure 7. Schematic presentation of directional recruitment. (A) In its DNA free form, the MBD and TRD of MeCP2 are not available for LANA interaction. (B) Upon binding to methylated DNA LANA binding surface in MeCP2 becomes available for LANA binding. (C) LANA tethers the viral episomal genomes to cellular chromosomes by binding to histones via its $\mathrm{N}$-terminal domain and to $\mathrm{MeCP} 2$ via its $\mathrm{C}$-terminal domain. This unidirectional recruitment of LANA by $\mathrm{MeCP} 2$ is essential for viral genome maintenance during latency. 